[Au: At this stage, l've edited the manuscript for brevity and clarity. Queries are meant to draw your attention to edits, inconsistencies or issues that are unclear. If we just ask you to confirm edits are correct, a simple yes/ok between the brackets will do [Au:OK? Is this what you meant? Edits OK? yes]. If questions are asked, please rephrase/update the manuscript text when addressing queries, so that the message is conveyed to the reader (please do not just type your answer to our query unless it is unclear).

As the reviewers noted, the manuscript is in very good shape and most comments and edits are fairly minor. However, there is an outstanding issue that must be addressed before we go forward. Figures 7 and 8 are currently referred to in the text in an alternating fashion (e.g. Figure 7A, 8A, 7B, 8B). This is against our style and might make that part of the manuscript difficult to follow for the reader owing to the need to refer to multiple figures at a time while reading the text. To clarify this issue, I recommend restructuring these figures. To do this, can you provide new chemdraw/PDFs with amended figures, including the following amendments:

a. Merge Figures 7A and $8 \mathrm{~A}$ to create a new 'Figure 7 ' that shows the methods of introducing $\mathrm{CF} 2 \mathrm{H}$ at sp3-carbons.

b. Merge figures $7 \mathrm{~B}$ and $8 \mathrm{~B}$ to make a new figure 8 that shows the main protocols of introducing $\mathrm{CF} 2 \mathrm{H}$ at heteroaromatic carbons. $7 \mathrm{C}$ could be shown in it's own small figure (Figure 9) or could be added as panel B in the new figure 8.

c. Amend the figure legends accordingly

Apologies for another figure change but this is essential to keep the figure callouts in style and I think it will make the figures much easier for the reader to follow. I've asked the art editor to delay redrawing these particular figures until we receive these updated chemdraws/PDFs. If there are any issues with this change, please do let me know as soon as possible and we can discuss further. 


\section{Contemporary synthetic strategies in organofluorine chemistry} Robert Britton ${ }^{1}$, Veronique Gouverneur ${ }^{2}$, Jin-Hong $\mathrm{Lin}^{3}$, Michael Meanwell ${ }^{1}$, Chuanfa
$\mathrm{Ni}^{3}$, Gabriele Pupo ${ }^{2}$, Ji-Chang Xiao ${ }^{3}$ and Jinbo $\mathrm{Hu}^{3, \dagger}$

${ }^{1}$ Department of Chemistry, Simon Fraser University, Burnaby, British Columbia, Canada.

${ }^{2}$ Chemistry Research Laboratory, University of Oxford, Oxford, U.K.

${ }^{3}$ Key Laboratory of Organofluorine Chemistry, Center for Excellence in Molecular Synthesis, Shanghai Institute of Organic Chemistry, University of Chinese Academy of Sciences, Chinese Academy of Sciences, Shanghai, China

†e-mail: jinbohu@sioc.ac.cn (Jinbo Hu)

\section{Author contributions}

Introduction (J.H.); Experimentation, Applications, Reproducibility and Data Deposition, and Limitations and Optimizations: Fluorination (G.P. and V.G.), Trifluoromethylation (J.-H.L. and J.-C.X.), Difluoromethylation (C.N. and J.H.), Monofluoromethylation (M.M. and R.B.); Outlook (J.H.); Overview of Primer (all authors). 


\section{ABSTRACT}

Fluorinated molecules have a wide range of applications and are used as medicines, agrochemicals and refrigerants and in smart phone liquid crystal displays, photovoltaic solar cells, Teflon tapes and the coatings of textiles and buildings [Au:OK? yes]. Fluorination and fluoroalkylation - incorporation of a trifluoromethyl, Formatted: Font color: Red difluoromethyl or monofluoromethyl group — and are the major strategies used for the construction of carbon-fluorine bonds and fluorinated carbon-carbon bonds, respectively. The last two decades have witnessed a rapid growth in fluorination and fluoroalkylation methods thanks to the development of new reagents and catalysts. This Primer aims to provide an overview of state-of-the-art strategies in fluorination, trifluoromethylation, difluoromethylation and monofluoromethylation, with an emphasis on using $\mathrm{C}-\mathrm{H}$ functionalization, although other strategies for fluorination and fluoroalkylation are also discussed. Further landmark achievements are expected in the fields of fluorination and fluoroalkylation as organofluorine compounds are used increasingly in everyday applications. [Au:OK? yes] 


\section{[H1] INTRODUCTION}

Fluorine is the most electronegative element in the periodic table and the second most used heteroelement [G] in life science research after nitrogen. ${ }^{1}$ Approximately $20 \%$ of marketed drugs and $50 \%$ of agrochemicals registered in the last 20 years are estimated to contain one or more fluorine atoms. ${ }^{2-7}$ Fluorine atoms can be incorporated through fluorination or fluoroalkylation, which introduction of a trifluoromethyl ( $\left.\mathrm{CF}_{3}\right)$, difluoromethyl $\left(\mathrm{CF}_{2} \mathrm{H}\right)$ or monofluoromethyl $\left(\mathrm{CH}_{2} \mathrm{~F}\right)$ group. The incorporation of fluorine atoms into a drug molecule can modulate several important properties including its metabolism, pharmacokinetics and ability to permeate biological tissues; as a result, the pharmaceutical industry relies on fluorination and fluoroalkylation methods and pressure is mounting to make these processes more environmentally friendly. ${ }^{2,8-10}$ In addition, because the unnatural fluorine-18 isotope possesses many desirable properties for applications in positron emission tomography [G] (PET) imaging, there is great demand for convenient methods enabling late stage $\left[{ }^{18} \mathrm{~F}\right]$ fluorination and $\left[{ }^{18} \mathrm{~F}\right]$ fluoroalkylation. ${ }^{11}$

Although inorganic fluorides such as $\mathrm{CaF}_{2}$ are abundant on earth, the organofluorine compounds typically required for the synthesis of pharmaceuticals are extremely rare in nature. ${ }^{12,13}$ As a result, almost all of the organofluorine compounds and materials used in industry and academia are synthetic [Au:OK? yes]. The source of fluorine for all fluorination and fluoroalkylation reactions is HF, which is prepared from the reaction of $\mathrm{CaF}_{2}$ mineral (also called fluorite or fluorspar) with sulfuric acid.

Fig. 1a outlines historical developments in organofluorine chemistry (FIG. 1a). The first chemically synthesized organofluorine compounds can be dated back to 1835 , when fluoromethane was prepared from dimethyl sulfate and KF. ${ }^{14,15}$ Over the past 200 years, many fluorination and fluoroalkylation methods have been developed to prepare structurally diverse organofluorine compounds. ${ }^{15-22}$ Before the 1960 s, fluorination mainly relied on corrosive or explosive reagents such as $\mathrm{HF}, \mathrm{F}_{2}, \mathrm{SbF}_{3}$ and $\mathrm{CoF}_{3}$ and classical reactions such as the Swarts reaction [G], the Balz-Schiemann 
reaction [G], the halex reaction [G], the Simons process (electrochemical fluorination) and direct fluorination ${ }^{23}$. Although these classical fluorination methods are inexpensive, they can be unsafe, require special equipment and offer low tolerance of common functional groups; as a result, they are only used in a small number of specialized chemical plants and research facilities. After the 1960s, more mild and selective fluorination reagents emerged, including diethylaminosulfur trifluoride (DAST), DeoxoFluor $^{\circledR}$, Selectfluor ${ }^{\circledR}$ and N-fluorobenzenesulfonimide (NFSI). Nucleophilic, electrophilic and radical fluoroalkylation reagents have also been developed, such as trifluoromethyltrimethylsilane ( $\mathrm{TMSCF}_{3}$, also known as Ruppert-Prakash reagent), methyl fluorosulfonyldifluoroacetate $\left(\mathrm{FSO}_{2} \mathrm{CF}_{2} \mathrm{CO}_{2} \mathrm{Me}\right.$, also known as Chen's reagent), sodium trifluoromethanesulfinate $\left(\mathrm{CF}_{3} \mathrm{SO}_{2} \mathrm{Na}\right.$, also known as Langlois reagent), and Umemoto and Togni reagents. Fluorination and fluoroalkylation reactions mediated or catalyzed by transition metals have improved the functional group tolerance of these methods and now enable the late-stage fluorination and fluoroalkylation of complex molecules. Synthetic organofluorine chemistry has flourished in the past 20 years with the development of many new fluorination and fluoroalkylation methods including enzymatic fluorination, enantioselective fluorination and fluoroalkylation, transition metal-catalyzed fluorination and fluoroalkylation, radical fluorination and fluoroalkylation, and ${ }^{18} \mathrm{~F}$ labelling of PET probes, among others. ${ }^{11,15,24-28}$

This Primer focuses on $\mathrm{C}-\mathrm{H}$ fluorination, trifluoromethylation, difluoromethylation and monofluoromethylation, and gives an introductory overview of other strategies for fluorination and fluoroalkylation (Fig. 1b), specifically discussing substrate scope, plausible mechanisms and the applications of these reactions for the synthesis of bioactive molecules and ${ }^{18} \mathrm{~F}$-labelled PET agents. When choosing examples to illustrate fluorination and fluoroalkylation chemistry, we have taken into account the recent guidance from industry that fluorination and fluoroalkylation methods should be safe, practical, cost-effective, environmentally responsible and capable of being performed in the presence of heterocycles, with a reasonable functional group 
119 tolerance. ${ }^{2}$ In the last part of this Primer, we briefly provide our outlook on the future of 120 fluorination and fluoroalkylation chemistry. 


\section{[H1] EXPERIMENTATION}

There have been great advances in the development of catalytic methods for the incorporation of single fluorine atoms or fluorinated groups into organic molecules over the past few decades. Many of these advances result from strategies aimed at optimizing reactivity and selectivity, for example combining judicious selection of fluorination and fluoroalkylation reagents with innovations in catalyst design. ${ }^{29,30}$ This section gives a brief introduction to fluorination with an emphasis on recently developed late-stage $\mathrm{C}-\mathrm{H}$ fluorination methods, before discussing trifluoromethylation, difluoromethylation and monofluoromethylation strategies.

\section{[H2] Fluorination}

Several highly efficient reagents have been developed for fluorination at both $s p^{2}$ and $s p^{3}$ carbons. The most prominent are novel nucleophilic reagents, including HF derivatives such as HF-pyridine, triethylamine trihydrofluoride $\left(\mathrm{Et}_{3} \mathrm{~N} \cdot 3 \mathrm{HF}\right)$ and $\mathrm{HF}$ complexed with 1,3-dimethyl-3,4,5,6-tetrahydro-2-pyrimidinone (DMPU);31-33 deoxyfluorinating reagents that gradually release fluoride in solution such as DAST, Deoxo-Fluor $^{\circledR}$, Phenofluor ${ }^{\mathrm{TM}}$ and PyFluor; ${ }^{34}$ hydrogen-bonded tetrabutylammonium fluoride (TBAF) [Au:OK? yes] complexes such as $\operatorname{TBAF}(t \mathrm{BuOH})_{4}$ and $\operatorname{TBAF}(\mathrm{pin})_{2}$ that are tuneable in terms of their nucleophilicity; ${ }^{35}$ and electrophilic reagents such as Selectfluor ${ }^{\circledR}$ and NFSI that can also serve as powerful oxidants. ${ }^{36}$

New catalytic manifolds have also played a critical role in augmenting fluorination approaches. Early studies focused on the use of pre-functionalised substrates and transition metal catalysis to overcome the problem of reductive elimination for $\mathrm{C}-\mathrm{F}$ bond formation; it is now clear that reductive elimination using $\mathrm{Pd}^{0} / \mathrm{Pd}^{\text {ll }}$ catalytic cycles is feasible with an bulky ancillary ligand that forces C-F bond formation from a 14electron Pd" complex. ${ }^{37}$ Additional advances were made with the realization that reductive elimination is energetically more facile using high oxidation metal complexes featuring in $\mathrm{Pd}^{\mathrm{II}} / \mathrm{Pd}^{\mathrm{IV}}$ and $\mathrm{Cu}^{\prime} / \mathrm{Cu}^{\mathrm{III}}$ catalytic cycles, and with the design of novel
Formatted: Font color: Blue Formatted: Font color: Red Formatted: Font: Italic 
ligands. Additional advances in the design of novel ligands were made following the discovery that reductive elimination is energetically more facile using high-oxidation metal complexes featuring in $\mathrm{Pd}^{H} / \mathrm{Pd}^{\mathrm{IV}}$-and $\mathrm{Cu}^{\mathrm{H}} / \mathrm{Cu}^{\mathrm{IH}}$-catalytic cycles. ${ }^{38-40}$ [Au:OK?

Please check intended meaning has been retained. No!!! Please use the original

Formatted: Font color: Red expression] Photoredox catalysis approaches have also emerged in recent years, for example those using decarboxylative fluorinations ${ }^{41,42}$.

Fluorination at $s p^{3}$ carbons can give rise to the challenge of controlling stereoselectivity and most enantioselective methodologies developed to date require electrophilic fluorination reagents and transition metal catalysis, or organocatalytic approaches such as enamine catalysis or cationic/anionic phase transfer catalysis [G] ${ }^{43-46}$. Recently, an alternative strategy inspired by the fluorinase enzyme and based on the merger between hydrogen bonding and phase transfer catalysis (HB-PTC) has allowed the use of cost-effective and easy-to-handle alkali metal fluorides as fluorine sources in asymmetric catalysis. ${ }^{47-49}$

The incorporation of fluorine through $\mathrm{C}-\mathrm{H}$ activation ${ }^{25}$ avoids the need to prefunctionalize the substrate. In principle, it allows for selective functionalization at a late stage in synthesis, which is highly desirable when preparing novel drugs and chemical libraries. ${ }^{50}$ To date, the field has been dominated by processes using electrophilic $\left(\mathrm{F}^{+}\right)$ sources [Au: Changed to match how $\mathrm{F}+$ is referred to in the paragraph below OK? yes] as these can act as both a fluorine source and oxidant; however, approaches have emerged that use a nucleophilic fluorine source in combination with a suitable external oxidant. $\mathrm{C}-\mathrm{H}$ fluorinations can be broadly divided into two classes depending on the activation mode employed: transition metal (TM)-catalyzed protocols, in which the metal is directly involved in the $\mathrm{C}-\mathrm{H}$ activation step, and radical-based methodologies, in which a carbon-centred radical is involved (FIG. 2A). 
[H3] Transition metal-catalyzed $\mathrm{C}-\mathrm{H}$ activation protocols

The main TM-catalyzed strategy uses a Pd"/IPdV catalytic cycle, which begins with the metal coordinating a directing group followed by metalation of a specific aromatic or aliphatic $\mathrm{C}-\mathrm{H}$ bond (FIG. 2B). Oxidation of the metal by an electrophilic $\left(\mathrm{F}^{+}\right)$source or an oxidant used in combination with a nucleophilic $\left(\mathrm{F}^{-}\right)$source ensures the generation of a high-valent species that undergoes $\mathrm{C}-\mathrm{F}$ bond formation through reductive elimination and regeneration of the catalyst. The conversion of aromatic $\mathrm{C}-$ $\mathrm{H}$ bonds to $\mathrm{C}-\mathrm{F}$ bonds can be performed on substrates bearing a 2-pyridinyl directing group, using $\mathrm{N}$-fluoropyridinium tetrafluoroborate as both an oxidant and fluorinating agent and $\mathrm{Pd}(\mathrm{OAc})_{2}$ as a catalyst ${ }^{38}$ (mechanistic studies have reported the involvement of a high-valent metal species for this reaction ${ }^{51}$ ). Several methodologies using a variety of directing groups in combination with $\mathrm{N}-\mathrm{F}$ reagents have now been described, expanding the scope of aromatic C-H fluorination (FIG 3A). ${ }^{25,52,53}$ An alternative approach uses a $\mathrm{Cu}^{\prime} / \mathrm{Cu}^{\text {III }}$ catalytic cycle ${ }^{39,40}$ and has been successfully translated to ${ }^{18} \mathrm{~F}$-radiolabeling (FIG $\left.3 \mathrm{~A}\right) \cdot{ }^{54}$

Aliphatic functionalization is more challenging than aromatic functionalization owing to the absence of a m-system for pre-coordination of the metal to the substrate and the greater conformational flexibility of aliphatic groups [Au:OK? yes]. Initial studies showed the feasibility of $\mathrm{C}\left(s p^{3}\right)-\mathrm{F}$ reductive elimination from high-valent $\mathrm{Pt} \mathrm{IV}^{\mathrm{V}}$ and $\mathrm{Pd}^{\mathrm{IV}}$ species. ${ }^{55,56}$ Subsequent reports proved that both bidentate and monodentate auxiliaries (FIG 3Ba) are suitable directing groups for the selective catalytic $\beta$ fluorination of amino acid derivatives using Selectfluor ${ }^{\circledR} .57,58$ A variety of additional carboxylic acid derivatives were successfully fluorinated by applying a similar strategy. ${ }^{25}$ Enantioselective $\mathrm{C}-\mathrm{H}$ fluorination can also be performed; asymmetric $\mathrm{C}\left(s p^{3}\right)-\mathrm{F}$ bond formation through $\mathrm{C}-\mathrm{H}$ activation has been achieved up to $96 \%$ ee using a chiral $\alpha$-amino acid derivative to form a transient chiral imine directing group and 2-alkyl benzaldehyde derivatives as substrates ${ }^{59,60}$. [Au:OK?_yes] A Formatted: Font color: Red mechanistically distinct $\mathrm{C}\left(s p^{3}\right)-\mathrm{H}$ fluorination on allylic substrates (FIG 3Bb) uses 
$\operatorname{Pd}(\operatorname{TFA})_{2}$ as a catalyst, a bis-sulfoxide ligand and a chromium-salen salt co-catalyst to generate the active $\mathrm{Pd}^{\prime \prime} \mathrm{m}$-allyl intermediate, which reacts with $\mathrm{Et} 3 \mathrm{~N} \cdot \mathrm{HF} .{ }^{6}$

[H3] Radical-based protocols

The main alternative to metal-catalyzed protocols for $\mathrm{C}-\mathrm{H}$ fluorination are protocols using carbon-centred radical intermediates. In these approaches, the absence of directing groups makes regioselectivity less predictable and reactivity follows general trends based on $\mathrm{C}-\mathrm{H}$ bond dissociation energies [G] (BDEs); consequently, only aliphatic, allylic and benzylic $\mathrm{C}-\mathrm{H}$ bonds are reactive. Polar and steric effects can also contribute to regioselectivity, with least substituted sites generally favoured.

Radical-based methodologies can be broadly categorized into non-photocatalytic and photocatalytic approaches. Non-photocatalytic approaches emerged following the discovery that reagents with low $\mathrm{N}-\mathrm{F}$ BDEs such as NFSI or Selectfluor ${ }^{\circledR}$ can be employed for fluorine atom transfer through the decarboxylative fluorination of peroxy esters. ${ }^{62}$ The first $\mathrm{C}-\mathrm{H}$ to $\mathrm{C}-\mathrm{F}$ conversion of aliphatic, allylic and benzylic substrates using carbon-centred radicals used a $\mathrm{Cu}^{\prime}$ initiator and $\mathrm{N}$-hydroxyphthalimide as a cocatalyst (FIG. 4A). ${ }^{63}$ Following this breakthrough, many other protocols involving metals such as silver and/or a variety of radical precursors were reported. ${ }^{25}$ Heteroaromatic benzylic $\mathrm{C}-\mathrm{H}$ bonds can be selectively fluorinated using a radical mechanism even in the absence of an external radical initiator when Selectfluor ${ }^{\circledR}$ is employed as a fluorine source ${ }^{64}$, and a complementary ionic approach to achieve the same transformation using NFSI has been proposed. ${ }^{65}$ Recently, a novel method has been reported for generating carbon-centred radicals for $\mathrm{C}-\mathrm{H}$ fluorination using formal

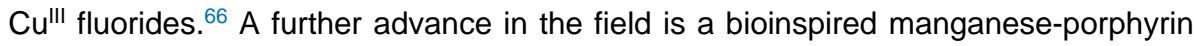


catalytic system for aliphatic $\mathrm{C}-\mathrm{H}$ fluorination using a nucleophilic source of fluoride $\mathrm{AgF}$ and $\mathrm{TBAF}$ - in the presence of $\mathrm{PhIO}$ as the oxidant (FIG. 4A). High regioselectivity was achieved using the above technique [Au: Using the above technique or a different system? Using the above technique] in the fluorination of complex steroids ${ }^{67}$ and later benzylic $\mathrm{C}-\mathrm{H}$ bonds. ${ }^{68}$ The nucleophilic fluorine source used in the above method can be used for ${ }^{18} \mathrm{~F}$-radiolabeling. ${ }^{69}$ Alternative nonphotocatalytic approaches ensure site selectivity by incorporating a specific functional group [Au:OK? yes] in the substrate that upon activation, enables a 1,5-hydrogen atom transfer process. These approaches generally feature Fell/Fell catalytic cycles and are applied to aliphatic and benzylic C-H fluorination reactions (FIG. 4B). ${ }^{25,70}$

Photocatalytic strategies use xanthones and 9-fluorenones as photocatalysts to enable the monofluorination and difluorination of benzylic $\mathrm{C}-\mathrm{H}$ bonds in the presence of Selectfluor ${ }^{\circledR}$ and visible light (FIG. 4C). ${ }^{71}$ This methodology sparked the broad use of aryl ketones and decatungstate salts as photocatalysts for radical $\mathrm{C}-\mathrm{H}$ fluorination ${ }^{25}$, including the elegant use of $\left[{ }^{18} \mathrm{~F}\right] \mathrm{NFSI}$ for site-selective radiolabelling of amino acids and peptides. ${ }^{72,73}$

[H3] Additional C-H fluorination methods

Additional mechanistically distinct methods for fluorination include the late-stage $\mathrm{C}-\mathrm{H}$ fluorination of pyridines and diazines under mild conditions using $\mathrm{AgF}_{2}$ for nucleophilic heteroaromatic substitutions ${ }^{74}$ and non-directed aromatic $\mathrm{C}-\mathrm{H}$ fluorination using a non-bound $\mathrm{Pd}^{\mathrm{II}}$ complex oxidized in situ to $\mathrm{Pd}^{\mathrm{IV}}$ by $\mathrm{F}^{+}$reagent ${ }^{75}$. In the latter method, fluoride delivery occurs through a transition state that density functional theory [G] (DFT) calculations have determined as a Pd ${ }^{\text {III }}$ singlet diradical. ${ }^{75}$ Both electron-rich and electron-poor arenes are tolerated, which is in stark contrast to uncatalyzed electrophilic aromatic fluorinations that require electron-rich arenes. Orthoregioisomeric and para-regioisomeric mixtures are obtained using this method. ${ }^{75}$
Formatted: Font color: Blue

Formatted: Font color: Red

Formatted: Font color: Red 


\section{[H2] Trifluoromethylation}

A number of trifluoromethylation reagents have been developed, such as $\mathrm{TMSCF}_{3}$ $\mathrm{FSO}_{2} \mathrm{CF}_{2} \mathrm{CO}_{2} \mathrm{Me}, \mathrm{CF}_{3} \mathrm{SO}_{2} \mathrm{Na}$, Togni reagent, Umemoto reagent and $\left[\mathrm{Ph}_{2} \mathrm{~S}^{+} \mathrm{CF}_{3}\right] \mathrm{TfO}$ (also known as Yagupolskii-Umemoto reagent). The emergence of these reagents has stimulated the development of various trifluoromethylation methods, including electrophilic, nucleophilic, radical and transition-metal catalyzed or mediated reactions (general reaction schemes shown in Fig. 5). ${ }^{76}$

[H3] Transition metal-catalyzed trifluoromethylation

Many transition metals have proven to be efficient at catalyzing trifluoromethylation reactions, [Au:OK? yes] including $\mathrm{Ni}^{77,78} \mathrm{Pd}^{79} \mathrm{Cu}^{80}$ and $\mathrm{Ag}^{81}$. $\mathrm{Cu}$ is the most commonly used transition metal catalyst for trifluoromethylation, owing to its high efficiency and low cost (Fig. 6A). Cu-promoted couplings always involve a $\mathrm{CuCF}_{3}$ complex, a key intermediate that can be generated in situ using reagent systems including: a Cu' source with $\mathrm{TMSCF}_{3}$ and a F- source, ${ }^{82-84}$ a $\mathrm{Cu}^{\prime}$ source with $\mathrm{FSO}_{2} \mathrm{CF}_{2} \mathrm{CO}_{2} \mathrm{Me}$; ${ }^{85-87}$ a $\mathrm{Cu}^{\prime}$ source with $(\mathrm{MeO})_{3} \mathrm{~B}^{-} \mathrm{CF}_{3} \mathrm{~K}^{+} ; 88 \mathrm{CuCl}$ with $\mathrm{HCF}_{3}$ and ${ }^{t} \mathrm{BuOK} ;{ }^{89,90}$ copper and $\left[\mathrm{Ph}_{2} \mathrm{~S}^{+} \mathrm{CF}_{3}\right] \mathrm{TfO}^{-; 91}$ and copper and Umemoto reagent ${ }^{92}$. $\mathrm{Cu}$ sources may be used in catalytic or stoichiometric quantities, depending on the reaction conditions. Some ligand-coordinated $\mathrm{CuCF}_{3}$ complexes are shelf-stable and can be used directly as reagents, including (phen) $\mathrm{CuCF}_{3}{ }^{93,94}$ and $\left(\mathrm{Ph}_{3} \mathrm{P}\right)_{3} \mathrm{CuCF}_{3}{ }^{95}$. Cupromoted coupling could be extended to a wide range of substrates, such as aryl/alkyl halides, aryl diazonium salts and aryl or alkyl boronic acids. It is generally accepted that reductive elimination from a $\mathrm{RCu}^{\text {III }} \mathrm{CF}_{3}$ complex is a key step in Cu-promoted coupling reaction; ${ }^{96-98}$ the exact mechanism for this step was elusive until mechanistic investigation of stable isolated $\mathrm{RCu}^{\mathrm{III}} \mathrm{CF}_{3}$ complexes revealed that reductive elimination proceeds through a concerted $\mathrm{C}-\mathrm{C}$ bond-forming pathway involving a threemembered-ring transition state. ${ }^{96-98}$ Electron-withdrawing ligands are favourable for 
this process as electrons flow from $\mathrm{R}$ and trifluoromethyl groups to the metal and ligands during reductive elimination.

[H3] Radical trifluoromethylation

Radical trifluoromethylation may occur through the addition of a $\mathrm{CF}_{3} \cdot$ radical into unsaturated functionalities (Fig. 6Ba) or the transfer of a $\mathrm{CF}_{3}$ group from $\mathrm{Cu}^{\prime \prime} \mathrm{CF}_{3}$ to an alkyl radical (Fig $6 \mathrm{Bb}$ ). The $\mathrm{CF}_{3} \cdot$ radical can be generated from various reagents, including $\mathrm{CF}_{3} \mathrm{SO}_{2} \mathrm{Na},{ }^{99} \mathrm{TMSCF}_{3},{ }^{100}$ Togni reagent, ${ }^{101,102}$ Umemoto reagent, ${ }^{101}$ $\left[\mathrm{Ph}_{2} \mathrm{~S}^{+} \mathrm{CF}_{3}\right] \mathrm{TfO}^{-},{ }^{101}$ and $\left.\mathrm{CF}_{3}\right|^{103}$ under oxidative or reductive conditions. Oxidants or reductants are usually required in stoichiometric quantities, although catalytic conditions can work if the redox reactions of the reagents can readily occur. The $\mathrm{CF}_{3}{ }^{\circ}$ radical can react with alkenes or alkynes to provide a variety of difunctionalized products. ${ }^{104,105}$ Trifluoromethylation of alkyl radicals has been much less developed. ${ }^{106}$ Alkyl radicals can be produced from alkyl halides, carboxylic acids and $\mathrm{C}-\mathrm{H}$ bonds, and the $\mathrm{Cu}^{\prime \prime} \mathrm{CF}_{3}$ complex may be generated in situ from $\mathrm{Cu}^{\prime \prime \prime} \mathrm{CF}_{3} /$ reductant or $\mathrm{Cu}^{\prime} \mathrm{CF}_{3} / \mathrm{oxidant}$. This radical process is limited to primary and secondary radicals; trifluoromethylation of tertiary radicals has not yet been achieved. It is unclear whether trifluoromethylation of the alkyl radical [Au: 'Trifluoromethylation of the alkyl radical' OK? Removed discussion of paths as this is covered in the figure. yes] involves the formation of a $\mathrm{Cu}^{\mathrm{III}} \mathrm{CF}_{3}$ intermediate or the direct transfer of the $\mathrm{CF}_{3}$ group from $\mathrm{Cu}^{\prime \prime} \mathrm{CF}_{3}$ to the alkyl radical without forming a $\mathrm{Cu}^{\prime \prime \prime} \mathrm{CF}_{3}$ intermediate (Fig. 6BC).

[H3] Nucleophilic and electrophilic trifluoromethylation

The direct nucleophilic attack of a $\mathrm{CF}_{3}{ }^{-}$anion on electrophiles is another strategy used for $\mathrm{CF}_{3}$ incorporation and $\mathrm{TMSCF}_{3}$ is the most commonly used reagent for this type of nucleophilic trifluoromethylation (Fig. 6C). ${ }^{107,108}$ The nucleophilic method can be extended to a wide range of electrophiles, including aldehydes, ketones, esters and imines. Electrophilic trifluoromethylation strategies can be applied to heteroatom [G] centered nucleophiles and carbon-centered nucleophiles (Fig. 6D). ${ }^{102}$ Carbon- 
centered nucleophiles must be electron-rich species, such as enolates or enamines, which can be generated in situ or prepared in advance. Electrophilic reagents include Umemoto reagent, ${ }^{109}$ Togni reagent, ${ }^{102}$ and Shibata reagent. ${ }^{110}$ Lewis acids can be used to enhance the electrophilicity of Togni reagent if the nucleophile is not reactive enough

[H3] C-H trifluoromethylation

$\mathrm{C}-\mathrm{H}$ trifluoromethylation is usually enabled by transition metal-catalyzed or transition metal-mediated methods for the metalation of a C$\mathrm{H}$ bond (Fig. 6Ea), or by radical-based approaches using a carbon-centered radical (Fig. 6Eb). Commonly used transition metals include Pd and Cu. Reductive elimination involves electron flow from a trifluoromethyl group to a metal; as the trifluoromethyl group is a strong electron-withdrawing group, reductive elimination is more facile when using a high-oxidation-state metal that can withdraw electrons more easily. Pdcatalyzed C-H trifluoromethylation usually involves a $\mathrm{Pd} / 1 / \mathrm{Pd}^{\mathrm{IV}}$ catalytic cycle, although a Pd"/Pd" cycle may also be involved. Pioneering work in Pd-catalyzed reactions used either pyridinyl, ${ }^{111}$ amide ${ }^{112}$ or amino moieties ${ }^{113}$ as directing groups for arene $\mathrm{C}\left(s p^{2}\right)$ $\mathrm{H}$ bond trifluoromethylation. For $\mathrm{Cu}$-catalyzed $\mathrm{C}-\mathrm{H}$ trifluoromethylation, a $\mathrm{Cu}^{\prime} / \mathrm{Cu}^{\prime \prime \prime}$ catalytic cycle is feasible. ${ }^{114,115}$ No directing group is required if the proton from the C$\mathrm{H}$ bond is acidic enough. ${ }^{114}$

Radical-based $\mathrm{C}-\mathrm{H}$ trifluoromethylation methods involve the generation of a radical from a $\mathrm{C}-\mathrm{H}$ bond through homolysis [Au:OK? yes]. ${ }^{116-118}$ Homolysis of alkyl Formatted: Font color: Red $\mathrm{C}-\mathrm{H}$ bonds is challenging owing to their high bond strength, and radical-based trifluoromethylation of alkyl $\mathrm{C}-\mathrm{H}$ bonds remains an active area of research. As described in Fig. 4Bc, the radical reaction proceeds through the transfer of a $\mathrm{CF}_{3}$ group - generated in situ from $\mathrm{Cu}^{\mathrm{ll}} \mathrm{CF}_{3}$ - to the alkyl radical.

Asymmetric catalytic trifluoromethylation remains largely unexplored. ${ }^{119-121}$ Reported asymmetric [Au:OK? yes] catalytic methods include nucleophilic, Formatted: Font color: Red electrophilic and radical trifluoromethylation using organocatalysts or transition-metal 
catalysis (FIG. 6F). Asymmetric nucleophilic reactions are limited to active electrophiles such as ketones and imines and asymmetric electrophilic and radical reactions require [Au:OK? yes] the generation of enolates or enamines from substrates. Further developments are needed for asymmetric catalytic trifluoromethylation as some pharmaceuticals such as the antiretroviral Efavirenz contain a $\mathrm{CF}_{3}-$ containing stereocenter andMild and general asymmetric catalytic trifluoromethylation approaches need to be further developed because current asymmetric catalytic trifluoromethylation methods have limited substrate scope. Additional examples of trifluoromethylations are shown in supplemental figure 1.

\section{[H2] Difluoromethylation}

Difluoromethyl compounds - organic compounds containing a $\mathrm{CF}_{2} \mathrm{H}$ group - are generally synthesized through difluorination ${ }^{122}$ or difluoromethylation ${ }^{123}$ reactions, hydrogenation of $\mathrm{gem}$-difluoroalkenes ${ }^{124}$ or hydrodefluorination of trifluoromethyl compounds. ${ }^{125}$ Difluoromethylation is the most straightforward of the above approaches owing to its good step economy and high functional group tolerance in introducing $\mathrm{CF}_{2} \mathrm{H}$ groups, and has progressed rapidly over the past decade. ${ }^{30,126-129}$ Here we introduce state-of-the-art methods for the formation of $\mathrm{C}\left(s p^{3}\right)-\mathrm{CF}_{2} \mathrm{H}$, (hetero)aryl- $\mathrm{CF}_{2} \mathrm{H}$ and heteroatom- $\mathrm{CF}_{2} \mathrm{H}$ bonds, all of which are of strong interest in pharmaceutical chemistry and have benefited from either reagent or catalyst design.

[H3] Difluoromethylation of $s p^{3}$ carbons

Difluoromethylated $s p^{3}$-carbon centers can be constructed via addition or substitution reaction of prefunctionalized substrates with a proper difluoromethylation reagent using prefunctionalized substrates and the addition of or substitution with a difluoromethylation reagent (FIG. 7A). The nucleophilic addition of a difluoromethanide
Formatted: Font color: Red

Formatted: Font color: Blue 
anion $\left(\mathrm{HCF}_{2}{ }^{-}\right)$or its equivalent $\left(\mathrm{FG}^{-\mathrm{CF}_{2}}{ }^{-}\right)$to electrophiles such as aldehydes, ketones and imines has been developed for the synthesis of difluoromethylated functional molecules such as alcohols and amines (FIG. 7Aa) and is normally achieved using either $\mathrm{PhSO}_{2} \mathrm{CF}_{2} \mathrm{H}$ or $\mathrm{TMSCF}_{2} \mathrm{H}$ as the reagent in the presence of a base (FIG. 8Aa).22,128,129,130,131 However, non-catalyzed nucleophilic substitution at sp $^{3}$-carbons is limited to the difluoromethylation of primary alkyl halides, such as $\mathrm{RCH}_{2} \mathrm{l}$, and pseudohalides, such as triflates. The use of metal catalysts allows for the difluoromethylation of a wide range of alkyl electrophiles. ${ }^{132-135}$ Recently, coppercatalyzed difluoromethylation of alkyl radicals has been developed, inspired by the trifluoromethylation of alkyl radicals (FIG. 7BAAb). This method proceeds through a $\mathrm{Cu}^{\prime} / \mathrm{Cu}^{\prime \prime} / \mathrm{Cu}^{\prime \prime \prime}$ catalytic cycle that involves single-electron transfer between $\mathrm{CuCF}_{2} \mathrm{H}$ species and redox-active alkyl electrophiles such as $N$-hydroxytetrachlorophthalimide (TCNHPI) esters and pyridinium salts (FIG. $8 \mathrm{Ab}) .{ }^{132,133}$ In the above case, the structurally well-defined zinc complex $(\mathrm{DMPU})_{2} \mathrm{Zn}\left(\mathrm{CF}_{2} \mathrm{H}_{2}\right.$ is the most suitable nucleophilic difluoromethyl source. This strategy allows the installation of a $\mathrm{CF}_{2} \mathrm{H}$ group at unactivated primary, secondary and even tertiary carbon centres. Addition of the difluoromethyl radical to alkenes represents a reliable method for the construction of $\mathrm{C}\left(s p^{3}\right)-\mathrm{CF}_{2} \mathrm{H}$ bonds under mild conditions (FIG. 7ㄷAc), ${ }^{128,129,136}$ which has recently stimulated the exploitation of structurally diverse, novel and practical difluoromethyl radical sources that can be readily activated under either chemical oxidation conditions or photoredox catalysis conditions-(FIG. $8 \mathrm{Ac}) .{ }^{137-140} \mathrm{C}-\mathrm{H}$ difluoromethylation at $s p^{3}$. carbon centers mainly focuses on the reaction of activated carbon acids with a difluorocarbene intermediate under the activation of a base (FIG. 7 DAd). ${ }^{141}$ The development of new difluorocarbene sources, including $\operatorname{TMSCF}_{2} \mathrm{Br}-(\mathrm{FIG}$. $8 \mathrm{Ad})$ and several S-(difluoromethyl)sulfonium salts [Au:OK? yes], has significantly improved reaction efficiency and greatly expanded substrate scope. ${ }^{128,129,142-144}$ The difluoromethylation of unactivated $\mathrm{C}-\mathrm{H}$ bonds is still rare (FIG. 7EEAe); however, Cucatalyzed benzylic $\mathrm{C}-\mathrm{H}$ difluoromethylation can proceed through the formation of
Formatted: Subscript

Formatted: Font color: Blue 
benzylic radicals after intramolecular $\mathrm{C}-\mathrm{H}$ activation, followed by the Cu-catalyzed transfer of difluoromethyl groups to the benzylic radicals (FIG. $8 \mathrm{Ae}) \cdot{ }^{145}$

[H3] (Hetero)aromatic difluoromethylation

(Hetero)aromatic difluoromethylation is a rapidly developing and practical method of synthesizing difluoromethyl (hetero)arenes (FIG. $\underline{87 B}$ ). ${ }^{126,127,30}$ These reactions can be categorized as: cross-couplings of organohalides or pseudohalides with $\mathrm{TMSCF}_{2} \mathrm{H},{ }^{146,147}$ structurally well-defined difluoromethylmetal complexes, ${ }^{148-152}$ or difluoromethyl radical sources (FIG. 8Aa-c7Ba and FIG. 8Ba-Bb);153,154 crosscouplings of organoboron or other organometallic reagents with difluoromethyl radical sources $^{155-158}$ or difluorocarbene sources ${ }^{159-162}$ (FIG. 8Ba-c7Bband FIG. 8BC-Bd); and C-H difluoromethylation with $\mathrm{TMSCF}_{2} \mathrm{H}^{163,164}$ or difluoromethyl radical sources ${ }^{165-170}$ (FIG. 8Ca-b7Be and FIG. 8Be). Difluoromethylation is more compatible with $\mathrm{Pd}, \mathrm{Ni}$ and Fe catalysis than trifluoromethylation, owing to the relative ease of the reductive elimination of heteroaryl- $\mathrm{CF}_{2} \mathrm{H}$ from the corresponding metal complexes. ${ }^{148,153,155}$ Difluoromethylation of aryl borons using Pd-difluorocarbene represents a novel method in this field that efficiently incorporates a $\mathrm{CF}_{2} \mathrm{H}$ group into an aromatic ring by using various difluorocarbene sources (FIG. $8 \mathrm{~B} \underline{c} \theta$ ), including the inexpensive industrial chemical $\mathrm{HCF}_{2} \mathrm{Cl}$ (also known as Freon-22, an ozone-depleting substance). ${ }^{159-162}$ As for fluorination and trifluoromethylation, the introduction of $\mathrm{CF}_{2} \mathrm{H}$ through $\mathrm{C}-\mathrm{H}$ bond activation is highly attractive for the late-stage modification of drug molecules and natural productsfunctional molecules. Available methods mainly rely on the innate nucleophilic reactivity of the $\mathrm{CF}_{2} \mathrm{H}$ radical towards heteroarenes. ${ }^{165-170}$ Strategies that offer different site-selectivities to direct radical difluoromethylation include the reaction of masked difluoromethyl radicals ${ }^{171,172}$ such as $\cdot \mathrm{CF}_{2} \mathrm{SO}_{2} \mathrm{Ph}$ with $\mathrm{CF}_{2} \mathrm{Cl}$, or the use of Cu-mediated oxidative C-H difluoromethylation and-with TMSCF $2 \mathrm{H}$ (FIG. 8들). ${ }^{163}$ [Au:OK? NO!!! "and" should read "with"] In addition, metal-catalyzed C-H functionalization of arenes followed by difluoromethylation offers a complementary approach for late-stage $\mathrm{C}-\mathrm{H}$ difluoromethylation. ${ }^{161,173,174}$ Vinylic difluoromethylation
Formatted: Font color: Blue

Formatted: Font color: Blue

Formatted: Font color: Blue

Formatted: Font color: Blue

Formatted: Font color: Blue
Formatted: Font color: Blue

Formatted: Font color: Red

Formatted: Font color: Red 
can be achieved, ${ }^{30,126-129}$ although this method has attracted less attention than (hetero)aromatic difluoromethylation.

[H3] Heteroatom difluoromethylation

Difluoromethylation of heteroatom nucleophiles with difluorocarbene is the most convenient method for obtaining functional molecules containing $\mathrm{HCF}_{2} \mathrm{O}, \mathrm{HCF}_{2} \mathrm{~S}$, and $\mathrm{HCF}_{2} \mathrm{~N}$ (FIG. 8D). ${ }^{174}$ The ozone-depleting substance $\mathrm{HCF}_{2} \mathrm{Cl}$ had been the most commonly used reagent for difluoromethylation of heteroatoms, although the. environmentally-benign alternatives $\mathrm{ClCF}_{2} \mathrm{CO}_{2} \mathrm{Na}, \mathrm{BrCF}_{2} \mathrm{P}(\mathrm{O})(\mathrm{OEt})_{2}, \mathrm{FSO}_{2} \mathrm{CF}_{2} \mathrm{CO}_{2} \mathrm{H}$ and $\mathrm{TMSCF}_{2} \mathrm{Br}$ are commercially available and frequently used. ${ }^{128,129,176}$ Unlike phenols, alcohols can react with difluorocarbene without deprotonation owing to the increased electron density of oxygen; $\mathrm{FSO}_{2} \mathrm{CF}_{2} \mathrm{CO}_{2} \mathrm{H}$ and $\mathrm{TMSCF}_{2} \mathrm{Br}$ are therefore suitable reagents for difluoromethylation of alcohols as they can release difluorocarbene under non-basic conditions. ${ }^{176,177}$ Recently, numerous methods for the incorporation of $\mathrm{HCF}_{2} \mathrm{~S}$ moiety have been developed to synthesize difluoromethyl thioethers. ${ }^{178}$

\section{[H2] Monofluoromethylation}

Few $\mathrm{C}-\mathrm{H}$ monofluoromethylation reactions had been reported to date, despite the ability of monofluoromethyl groups to serve as bioisosteres [G] for a variety of pharmaceutically relevant functional groups (for example, $\mathrm{CH}_{3}, \mathrm{CH}_{2} \mathrm{OH}$ and $\mathrm{CH}_{2} \mathrm{NH}_{2}$ ). ${ }^{22,27,123,179}$ A conventional two-step approach to monofluoromethylation involves an initial chloromethylation step known as the Blanc reaction, which uses formaldehyde and hydrogen chloride or zinc chloride to install a monochloromethyl functional group onto arenes and heteroarenes. ${ }^{180}$ Well-established halide-exchange reactions using fluoride salts such as $\mathrm{KF}$ or $\mathrm{AgF}$ support conversion to the 
monofluoromethyl derivative. ${ }^{181}$ An increased interest in $\mathrm{CH}_{2} \mathrm{~F}$ has inspired the development of several nucleophilic ${ }^{182-185}$, electrophilic ${ }^{186}$ and radical $^{187-189}$ monofluoromethylating reagents (FIG. 9A). The first example of electrophilic $\mathrm{C}-\mathrm{H}$ monofluoromethylation was reported in 1953 and involved the reaction of arenes with fluoromethanol in the presence of zinc (II) chloride, which afforded fluoromethylarenes. ${ }^{190,191} \mathrm{C}-\mathrm{H}$ monofluoromethylation reactions now [Au:OK? ves] primarily involve deprotonation of 1,3-dicarbonyls, followed by reaction of the resultant carbanion with an electrophilic monofluoromethylation reagent (FIG. 9B). ${ }^{192-195}$ For example, $\beta$-ketoesters can react with fluoroiodomethane and lithium tert-butoxide to produce $\quad$ a-monofluoromethyl adducts. ${ }^{193}$ Commonly, competitive monofluoromethylation is a complicating factor and in a few cases it is a predominant reaction pathway ${ }^{196}$ that is proposed to occur via a radical process. ${ }^{197,198}$ In this example, competitive O-monofluoromethylation, which is proposed to occur via a radical process ${ }^{197,198}$, is a complicating factor and in a few cases the-[Au:OK? No!!!]

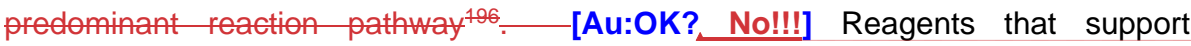
ehemoselective C-monofluoromethylation while avoiding the use of the ozonedepleting reagents chlorofluoromethane (CFC-31) and bromofluoromethane (CFC31B1) are needed. ${ }^{179} S$-(monofluoromethyl)diarylsulfonium tetrafluoroborate has been developed as an electrophilic source of $\mathrm{CH}_{2} \mathrm{~F}$ and supports the $\alpha$ monofluoromethylation of bis(phenylsulfonyl)methane and diethylmalonate derivatives (FIG. 9B). ${ }^{194}$ Subsequent work has also described the use of sulfonium ylides for the monofluoromethylation of 2-aryl-substituted and 2-alkyl-substituted malonates. ${ }^{195}$

Although the $\mathrm{CH}_{2} \mathrm{~F}$ radical was first observed and characterized in $1971,{ }^{199}$ its use for radical C-H monofluoromethylation was not demonstrated until 2012, when a series of zinc sulfinate salts were reported to be capable of transferring fluoroalkyl radicals to nitrogen-containing heteroarenes through a Minisci reaction [G] -like process (FIG. 9C). ${ }^{200}$ Among these zinc sulfinate reagents was zinc monofluoromethylsulfinate (MFMS), which reacts with a small collection of heteroarenes with excellent
Formatted: Font color: Red

Formatted: Font color: Blue 
regioselectivity. MFMS can be synthesized in four simple steps from 4-chlorobenzyl mercaptan on the scale of grams; however, unlike other zinc and sodium fluoroalkylsulfinates, it is not commercially available. The fluoroacetate radical, generated from a xanthate precursor, can also engage in a Minisci-like reaction with caffeine (FIG. 9C). ${ }^{201}$ Subsequent in situ decarboxylation of the carboxyfluoromethylated caffeine reveals a monofluoromethyl derivative. Further development of fluoroacetates as synthetic surrogates for the monofluoromethyl group has been independently reported 202,203 $^{2}$ FIG. 9D); for example, ruthenium-catalyzed, meta-selective $\mathrm{C}-\mathrm{H}$ monofluoromethylations of arenes have been accomplished using bromofluoroacetate. These reactions proceed via ortho $\mathrm{C}-\mathrm{H}$ insertion directed by a nitrogen-containing heteroaryl group heteroaromatic compound [Au:OK? No!!! "heteroaromatic compound" should read "heteroaryl group"] and subsequent nucleophilic addition of the fluoroacetate radical into the arene. Under photoredox copper catalysis conditions, fluoromethylsulfonyl chloride can be used as a source of monoflouromethyl radicals for additions into electron-deficient alkenes (FIG. 9E). ${ }^{188}$ Similarly, a ruthenium photoredox catalyst can be used for generating monofluoromethyl radicals from monofluoromethyl sulfones for radical additions into isocyanides. ${ }^{187}$ As a complimentary approach for addition of the monofluoromethyl group, nucleophilic reagents such as lithium anion (FIG 9F) have been used for the synthesis of terminal fluoroalkenes, ${ }^{185,204}$ 2,2-disubstituted fluorovinyl sulfones, ${ }^{183,184}$ the addition into ketones and imines ${ }^{182}$ and cross-coupling reactions. ${ }^{205}$

\section{[H2] Safety}

Safety is an important issue in synthetic organofluorine chemistry. In general, classical fluorination reagents such as anhydrous hydrogen fluoride $(\mathrm{HF})$, elemental fluorine $\left(\mathrm{F}_{2}\right)$ and sulfur tetrafluoride $\left(\mathrm{SF}_{4}\right)$ are highly corrosive, toxic and sometimes even explosive chemicals. When handling these dangerous reagents, protective equipment such as chemical splash goggles, acid-resistant apron, and gloves should be used and $21 / 62$
Formatted: Font color: Blue

Formatted: Font color: Blue

Formatted: Font color: Red 
experiments should be performed under good ventilation conditions. Glassware should be avoided as these corrosive fluorination reagents readily etch glass. A fume hood fitted with a scrubbing system should be used when handling large quantities of aHF as anhydrous and even dilute aqueous HF solutions are corrosive and rapidly damage tissues, owing to resorptive binding of fluoride to calcium and magnesium ions Antidotes such as calcium gluconate gel should be kept on-hand for primary treatment of HF burns of the skin. ${ }^{206}$

Modern fluorination and fluoroalkylation reagents are much milder and less dangerous than their classical counterparts. For most of the synthetic methods described in this Primer, experiments can be carried out in a standard organic chemistry laboratory and the reaction setup is generally not different from other organic reactions. However, some fluorination reagents such as $\mathrm{HF}-$ pyridine, $\mathrm{HF}-3 \mathrm{Et}_{3} \mathrm{~N}$ and DAST are corrosive and precautions should be taken when handling these chemicals. For ${ }^{18} \mathrm{~F}$-labeled synthesis, a standard radiochemistry laboratory for handling radioactive chemicals is required.

\section{[H1] APPLICATIONS}

In this section, we provide an introductory overview of how the above fluorination and fluoroalkylation methods are currently used in life-science related areas (Fig. 10). We initially focus on the application of these methods to drug discovery and development, including the late-stage modification of approved drugs or bioactive molecules and the synthesis of novel drug candidates. We then discuss the recent field of $\left[{ }^{18} \mathrm{~F}\right]$-radiolabeling, the products of which are used for disease diagnosis, treatment monitoring and mechanism research. Finally, we introduce the application of these methods to bioorganic chemistry research and the pharmaceutical and agrochemical industries. 


\section{[H2] Drug discovery}

More than 300 fluorinated drugs have been developed and used worldwide following the introduction of fludrocortisone as the first fluorine-containing pharmaceutical drug in $1954 .^{207}$ Fluorination of drug molecules can enhance their efficacy by modulating their absorption, distribution, metabolism, and excretion (ADME) profile and fluorination and fluoroalkylation have therefore become a routine and powerful tool in drug discovery. . $^{3-6,8-10,13,26}$

\section{[H3] Fluorination}

$\mathrm{C}-\mathrm{H}$ fluorination and ${ }^{18} \mathrm{~F}$-radiofluorination are at an early stage of research and their use in the late-stage functionalization of drug molecules in the context of drug discovery is still in its infancy, owing to numerous challenges such as difficulties in separating fluorinated derivatives from precursor molecules and achieving high chemoselectivity, regioselectivity and stereoselectivity. ${ }^{50}$ Despite these challenges, these techniques have been successfully used to synthesize fluorinated analogues of a handful of natural products and FDA-approved pharmaceuticals. In 2012, oxidative aliphatic $\mathrm{C}-\mathrm{H}$ fluorination using nucleophilic fluoride was applied to the selective fluorination of terpenoids such as sclareolide and steroids such as $5 \alpha$-androstan-17one. ${ }^{67}$ [Au:OK? NO!!! We have made a modification.] Fluorinated analogues of these natural products have also been accessed through a complementary radical-based approach using a decatungstate photocatalyst. ${ }^{208}$ The selective benzylic fluorination of dihydrocoumarin - a scaffold commonly found in bioactive natural products and drugs - was reported using a radical-based strategy 63 ; $\mathrm{C}-\mathrm{H}$ fluorination of sugar-derivatives was demonstrated using a site-selective and diastereoselective Pd"-catalyzed process (FIG. 10B1). ${ }^{57}$ Further, fluorinated variants of the antidiabetic Pioglitazone and Roflumilast - a drug used to treat chronic obstructive pulmonary disease - were synthesized through selective ortho fluorination of pyridines with $\mathrm{AgF}_{2}$ (FIG. 10A2). ${ }^{74}$
Formatted: Font color: Blue, Superscript

Formatted: Font color: Blue 
[H3] Trifluoromethylation

As a bioisostere of a chloride or methyl group, the trifluoromethyl group is often used to adjust the steric and electronic properties of a biologically active molecule or to protect a reactive methyl group from metabolic oxidation. ${ }^{209,210}$ Various trifluoromethylation methods have been widely used for the synthesis of $\mathrm{CF}_{3}$ containing biologically active molecules in the development of drugs and agrochemicals. The Cu-promoted coupling reaction of aryl or alkenyl halides is one of the preferred strategies for the construction of a $\mathrm{Csp}^{2}-\mathrm{CF}_{3}$ bond owing to its low cost, high site-selectivity and the convenience of upscaling. The compound GNE-7915 - a potent, selective, metabolically stable and brain-penetrant small molecule inhibitor of leucine-rich repeat kinase $2\left(\mathrm{LRRK}_{2}\right)^{211}$ — contains a $\mathrm{CF}_{3}$-pyrimidine motif that can be constructed through a Cu-promoted coupling reaction on a molar scale (FIG. 10A1). Alkyl- $\mathrm{CF}_{3}$ moieties may also be installed using other trifluoromethylation methods; for example, nucleophilic trifluoromethylation was used to synthesize a G-protein coupled receptor 40 (GPR40) agonist, in which the $\mathrm{CF}_{3}$ group increases the Ki of the drug with respect to its binding to human GPR40 and improves agonist efficacy (FIG. 10A3). ${ }^{212}$

[H3] Difluoromethylation

$\underline{\mathrm{CF}_{2}} \underline{\mathrm{H}}$ is a lipophilic bioisostere of hydroxyl and methyl groups, and can serve as a weak hydrogen-bond donor, therefore it can be used to improve the binding ability or metabolic stability of biologically active molecules. ${ }^{210} \mathrm{GF}_{2} \mathrm{H}$ is a more lipophilic bioisostere of hydroxyl and methyl groups than the CF 3 group [Au:OK? No!!! We have made a modification.], which can serve as a hydrogen-bond donor and therefore improve the binding ability of biologically active molecules. ${ }^{205}$ [Au:OK?No!!! We have made a modification.] Many recently developed carbon difluoromethylation methods have shown potential for the late-stage modification of pharmaceuticals and complex molecules, both through C-H difluoromethylation ${ }^{133,140,162,165,167,174,177,213-216}$ and the substitution of prefunctionalized groups such as halogen, ${ }^{147,149,153}$ carboxyl, ${ }^{132,151}$ hydroxyl ${ }^{162}$ and amino groups ${ }^{145,150}$ with $\mathrm{CF}_{2} \mathrm{H}$. Direct $\mathrm{C}-\mathrm{H}$ radical difluoromethylation of heterocycles with difluoromethanesulfinate salts is arguably the most significant 
achievement in this field; together with alkanesulfinate chemistry, this technique has found immediate application in drug discovery owing to its simplicity, the high tolerance towards functional groups and the predictability of site selectivity. ${ }^{217}$ The introduction of a $\mathrm{CF}_{2} \mathrm{H}$ group into deoxyuridine with $\mathrm{HCF}_{2} \mathrm{SO}_{2} \mathrm{Na}$ has led to the discovery of 2deoxy-5-difluoromethyluridine (F2TDR) (FIG. 10A4), a difluorinated analogue of the FDA-approved cancer drug trifluridine with an enhanced capacity to inhibit tumor cell proliferation [Au: Changed 'tumor cell inhibition' to 'to inhibit tumor cell proliferation' - OK? By tumour cell inhibition did you mean inhibition of tumour cell proliferation? yes]. . $^{167}$

[H3] Monofluoromethylation

Existing examples of $\mathrm{CH}_{2} \mathrm{~F}$-containing molecules are typically made through classical nucleophilic substitution chemistry. To date, contemporary $\mathrm{C}-\mathrm{H}$ monofluoromethylation reactions have not been reported in drug development programs, aside from a single demonstration of the use of fluoroiodomethane and base for the electrophilic C-H monofluoromethylation of androstane derivatives. ${ }^{192}$

\section{[H2] Radiolabeling}

The translation of fluorination to radiofluorination and the use of ${ }^{18} \mathrm{~F}$-containing molecules in PET represents a major application for fluorination techniques. ${ }^{218}$ [Au:OK? yes] PET is a powerful imaging technique that uses positron-emitting radiotracers to gather quantitative information on metabolic processes, allowing for the early diagnosis of diseases such as cancer [Au:Added some context here - OK? yes]. PET requires body and be detected by the PET scan. The radioisotope ${ }^{18} \mathrm{~F}\left(\mathrm{t}_{1 / 2}=109\right.$ minutes) is ideal for use in PET radiopharmaceuticals as it has a clean positron emission profile and its maximal positron energy is well-suited for high-resolution imaging. For these reasons, radiochemists are invested in developing new methodologies to efficiently produce ${ }^{18} \mathrm{~F}$-containing radiopharmaceuticals. 
[H3] [ $\left.{ }^{18} \mathrm{~F}\right]$ fluorination

$\mathrm{C}-\mathrm{H}^{18} \mathrm{~F}$-radiofluorination is an emerging field with only a few existing examples.

${ }^{18} \mathrm{~F}$ variants of natural products and some top-selling drug derivatives were elegantly accessed using a variant of the method used for Mn ${ }^{\text {III-catalyzed }}$ nucleophilic ${ }^{19} \mathrm{~F}$-fluorination. ${ }^{69}$ Some reports have also accessed ${ }^{18} \mathrm{~F}$ radiotracers through late-stage $\mathrm{C}-\mathrm{H}$ fluorinations, including in the automated synthesis of the retinoic acid receptor beta agonist $\left[{ }^{18} \mathrm{~F}\right] \mathrm{AC} 261066$ - which employed a Cul promoter (FIG. 10A5) ${ }^{54}$ - and radical-based ${ }^{18} \mathrm{~F}$ labeling of leucine derivatives (FIG. 10A6). ${ }^{219}$

[H3] [18 F]trifluoromethylation

Most radiofluorination efforts have focused on the development of ${ }^{18} \mathrm{~F}$ trifluoromethylation methods to support PET imaging experiments in animal models. The coupling of a $\left[\mathrm{CuCF}_{2}{ }^{18} \mathrm{~F}\right.$ ] complex is the most commonly used strategy for $\mathrm{CF}_{2}{ }^{18} \mathrm{~F}$ installation. The $\left[\mathrm{CuCF}_{2}{ }^{18} \mathrm{~F}\right]$ complex could be generated in situ from the combination of a $\mathrm{Cu}^{\prime}$ salt, a difluorocarbene $\left(: \mathrm{CF}_{2}\right)$ source and $\left.{ }^{18} \mathrm{~F}\right]$ fluoride ${ }^{220}$ or the combination of a $\mathrm{Cu}^{\prime}$ salt, preformed $\left.{ }^{18} \mathrm{~F}\right]$ fluoroform $\left(\mathrm{HCF}_{2}{ }^{18} \mathrm{~F}\right)$ and a base ${ }^{221-223}$. Recently, a : $\mathrm{CF}_{2} /{ }^{18} \mathrm{~F}^{-}$system was used to develop a Langlois-type ${ }^{18} \mathrm{~F}$ labeling reagent $\left(\mathrm{CF}_{2}{ }^{18} \mathrm{FSO}_{2} \mathrm{NH}_{4}\right)$ that enables radical $\mathrm{C}-\mathrm{H}\left[{ }^{18} \mathrm{~F}\right]$ trifluoromethylation of aromatic residues in peptides. ${ }^{224}$ Interestingly, the same research group also developed a fully automated radiosynthesis of octreotide[Trp $\left.\left(2-\mathrm{CF}_{2}{ }^{18} \mathrm{~F}\right)\right]$ using the Advion NanoTek microfluidic synthesis system (FIG. 10 A8). ${ }^{224}$ A preliminary in vivo PET imaging experiment using this labeling peptide on naïve rats suggested this complex is cleared from the body by urinary excretion and through the gastrointestinal tract, facilitating the measurement of the distribution and pharmacokinetics of octreotide in vivo.

[H3] [18F]difluoromethylation

Direct $\left[{ }^{18} \mathrm{~F}\right]$ difluoromethylation is more challenging than direct $\left[{ }^{18} \mathrm{~F}\right]$ trifluoromethylation, owing to the difficulty in constructing the $\left[{ }^{18} \mathrm{~F}\right]$ difluoromethylation reagent. Inspired by the radical difluoromethylation reagent $26 / 62$
Formatted: Font color: Blue

Formatted: Font color: Blue, Superscript

Formatted: Font color: Blue

\section{Formatted: Font color: Blue, Subscript}

Formatted: Font color: Blue

Formatted: Font color: Blue 
difluoromethyl 2-benzo-[d]thiazolyl sulfone $\left(2-\mathrm{BTSO}_{2} \mathrm{CF}_{2} \mathrm{H}\right),{ }^{187}$ a new reagent, 2$\mathrm{BTSO}_{2} \mathrm{CF}^{18} \mathrm{FH}$, was prepared in two steps and successfully applied for $\mathrm{C}-\mathrm{H}$ $\left[{ }^{18} \mathrm{~F}\right]$ difluoromethylation of a broad range of $\mathrm{N}$-heteroaromatics, including drug molecules such as the antiviral medication Acyclovir (FIG. 10A7). ${ }^{213}$

[H3] [18F]monofluoromethylation

No examples of $\mathrm{C}-\mathrm{H}\left[{ }^{18} \mathrm{~F}\right]$ monofluoromethylation have been reported thus far, although $\left[{ }^{18} \mathrm{~F}\right]$ fluoromethylhalides have been prepared and used in the electrophilic $\left[{ }^{18} \mathrm{~F}\right]$ fluoromethylation of $\mathrm{N}$-nucleophiles, $\mathrm{O}$-nucleophiles and $\mathrm{S}$ - nucleophiles for their potential application in PET imaging. ${ }^{225-227}$ Additionally, $\left[{ }^{18} \mathrm{~F}\right] \mathrm{FCH}_{2} \mathrm{X}$ reagents (where $\mathrm{X}=\mathrm{Br}$ or I) have been used in palladium-catalyzed cross-couplings with boronic acids to generate ${ }^{18} \mathrm{~F}$-radiolabeled (hetero)arenes. ${ }^{228}\left[{ }^{18} \mathrm{~F}\right]$-fluoromethylhalides are readily synthesized from $\mathrm{CH}_{2} \mathrm{X}_{2}(\mathrm{X}=\mathrm{I}$ or $\mathrm{Br}$ ) through halide-exchange reactions with a $\left[{ }^{18} \mathrm{~F}\right] \mathrm{fluoride}$ salt; ${ }^{225-227}$ for example, in acetonitrile at reflux the halide-exchange ${ }^{226}$ of dibromofluoromethane with $\left[{ }^{18} \mathrm{~F}\right] \mathrm{KF}$ and Kryptofix $222-\mathrm{a}$ cryptand $[\mathrm{G}]$ that complexes potassium - affords $\left[{ }^{18} \mathrm{~F}\right] \mathrm{FCH} 2 \mathrm{Br}$ at an excellent radiochemical yield $(47 \pm 8 \%, \mathrm{n}=$ 20). ${ }^{226}$ Furthermore, a second halide-exchange reaction of $\left[{ }^{18} \mathrm{~F}\right] \mathrm{FCH} 2 \mathrm{Br}$ with silver triflate delivers the corresponding ${ }^{18} \mathrm{~F}$-radiolabeled triflate. These established procedures should inspire the use of $\mathrm{C}-\mathrm{H}\left[{ }^{18} \mathrm{~F}\right]$ monofluoromethylation approaches and the development of new $\left[{ }^{18} \mathrm{~F}\right]$ monofluoromethylation reagents. Notably, $\approx 60 \%$ of all FDA approved drugs contain at least one heterocycle, with pyridine being among the most common ${ }^{229}$; thus, the preparation of $\left[{ }^{18} \mathrm{~F}\right] \mathrm{MFMS}$ would be of particular use for radiochemistry owing to its demonstrated regioselectivity when deriving medicinally relevant heterocycles, including pyridine and purine ring systems. Such an approach will require a marked reduction in the time required for the synthesis of MFMS ( 4 days) for it to be compatible with the $t_{1 / 2}$ of ${ }^{18} \mathrm{~F}(\sim 110 \mathrm{~min})$. 


\section{[H2] Bioorganic chemistry research}

Radical C-H fluoroalkylation of native aromatic and heteroaromatic residues in peptides or proteins can facilitate their study using ${ }^{19} \mathrm{~F}$ NMR analysis and potentially modulate their functions. Fluoroalkanesulfinate salts are the reagents of choice owing to their biocompatibility and the mildness of their reaction conditions. ${ }^{217}$ Selective trifluoromethylation of tyrosine (Tyr) residues in recombinant human insulin ${ }^{230}$ and tryptophan (Trp) residues in the enzyme lysozyme (FIG. 10B2) ${ }^{231}$ have been achieved with $\mathrm{CF}_{3} \mathrm{SO}_{2} \mathrm{Na}$. Trifluoromethylation modification of peptides and proteins provides a sensitive ${ }^{19} \mathrm{~F}$ NMR spectroscopic probe of the local environment in the biomacromolecules, while minimally perturbing their overall structure and enzymatic function.

The application of difluoromethylation reactions in biological research has been showcased by the development of a novel quinone methide-based, self-immobilizing fluorogenic probe that allows the measurement of $\beta$-galactosidase activity in living cells (FIG. 10B3). ${ }^{216}$ Nucleophilic attack of a $\mathrm{CF}_{2} \mathrm{H}$ group on the coumarin group of the probe by $\beta$-galactosidase activates fluorescence in the molecule and allows measurement of enzyme activity. The 3-difluoromethylated analogue of the probe, obtained through the one-step reaction of coumarin with $\mathrm{HCF}_{2} \mathrm{SO}_{2} \mathrm{Na}$, was shown to have a higher fluorogenic response and improved fluorescence labeling efficiency over a previously developed 8-difluoromethylated analogue. ${ }^{216}$

\section{[H2] Pharmaceutical and agrochemical production}

In the production of pharmaceuticals and agrochemicals, fluorine and fluoroalkyl groups are usually introduced to intermediates at the early stage of the synthesis through the fluorination of prefunctionalized precursors or transformation of fluoroalkylcontaining building blocks, respectively.207,7 An exception is electrophilic difluoromethylation with difluorocarbene, which has been widely applied in early-stage 


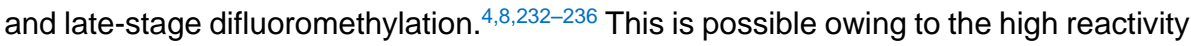
of difluorocarbene towards heteroatom nucleophiles and soft carbon nucleophiles and the low cost of difluorocarbene sources such as $\mathrm{HCF}_{2} \mathrm{Cl}$. Pharmaceuticals and agrochemicals synthesized using this electrophilic difluoromethylation method include the aforementioned anti-pulmonary disease drug Roflumilast FIG. 10A2), ${ }^{4}$ Pantoprazole - a drug employed for the treatment of gastroesophageal reflux disease $^{8}$ - the herbicide Pyraflufen-ethyl (FIG. 10B4), ${ }^{234}$ and the insecticide Pyriprole (FIG. 10B5), ${ }^{236}$ among others.

\section{[H1] REPRODUCIBILITY AND DATA DEPOSITION}

\section{[H2] Reproducibility}

All of the fluorination and fluoroalkylation methods highlighted in this Primer should be reproducible by virtue of the fact that they have been published in peer-reviewed journals with detailed experimental procedures and adequate analytical and characterization data. However, most methods have emerged in the last 10-15 years and have only been demonstrated on small ( $\mathrm{mmol}$ ) scales. More studies will be needed to fully evaluate reproducibility issues and assess the potential of these synthetic methods following process scale-up. For any given transition-metal catalyzed fluorination or fluoroalkylation reaction, the commercially available starting materials are used as received from the laboratory chemical suppliers and organic solvents are pre-dried to remove trace water, unless otherwise noted in the original literature. The reaction is usually conducted using standard laboratory equipment under the protection of an inert atmosphere, such as argon or nitrogen.

For nucleophilic fluoroalkylations using fluoroalkylmetal reagents that are in-situ generated by deprotonation or halogen-lithium exchange, low temperatures (such as $-78{ }^{\circ} \mathrm{C}$ ) are required to avoid thermal decomposition. ${ }^{22}$ The radical $\mathrm{C}-\mathrm{H}$ fluoroalkylation of (hetero)aromatics with fluoroalkanesulfinate salts can be performed 
under open air without an organic co-solvent and in the presence of extensive impurities in the reaction medium; however, portion-wise addition of large excess amounts of the reagents are usually needed to achieve a high yield. ${ }^{217}$

The synthesis of PET tracers requires high levels of reproducibility and applicability to biological studies and clinical applications. ${ }^{237}$ These syntheses should be efficient (non-decay corrected radiochemical yield of 5-40\%) and timeframes should be compatible with the short $t_{1 / 2}$ of ${ }^{18} \mathrm{~F}(\sim 110 \mathrm{~min})$. Products should be manufactured using automated synthesis, obtained in high radiochemical purity, must have sufficient specific radioactivity for imaging $(\geqslant 8 \mathrm{Mbq} / \mathrm{kg}$ ) and must be produced in a form acceptable to regulatory bodies. ${ }^{238}$ The latter requirement is valid for all pharmaceuticals and involves using good manufacturing practices (GMP) so that clinical products are produced consistently and controlled according to quality standards. GMP covers all aspects of production including raw materials, equipment and personnel training. Different countries and authorities have their own GMP guidelines aiming to ensure high reproducibility, quality and the safety of the finished product. $^{239}$

Following the general standard for reporting organic synthesis, all new small molecule chemical products should be characterized using ${ }^{1} \mathrm{H}$ NMR and ${ }^{13} \mathrm{C}$ NMR spectroscopy, and high-resolution mass spectroscopy analysis, with results reported in publications, either in the main text or in the electronic supporting information. ${ }^{19} \mathrm{~F}$ NMR data should also be provided to support the incorporation of the desired fluorinated moieties. Inexperienced chemists can refer to an elegant book on fluorine NMR to interpret the ${ }^{1} \mathrm{H},{ }^{13} \mathrm{C}$ and ${ }^{19} \mathrm{~F}$ NMR data of organofluorine compounds. ${ }^{240}$ In the case of ${ }^{18} \mathrm{~F}$-labelling, the ${ }^{19} \mathrm{~F}$-substituted analogue should be synthesized as a standard sample for characterization and high performance liquid chromatography analysis. For the modification of biomacromolecules, ${ }^{19} \mathrm{~F}$ NMR yield is used to assess the efficiency of fluorine incorporation. [Au: Removed sentence on reproducibility as this is mentioned at the beginning of this section. OK!] 


\section{[H1] LIMITATIONS AND OPTIMIZATIONS}

\section{[H2] Fluorination}

The direct fluorination of $\mathrm{C}-\mathrm{H}$ bonds represents an ideal solution for late-stage fluorinations; however, it also presents unique challenges. ${ }^{50}$ The similar steric parameters and chromatographic behaviour of the precursor and the fluorinated product can cause purification issues if the reaction does not reach full conversion. In many cases, the product has a [Au:OK? yes] reactivity profile similar to the starting material; preventing polyfluorination is therefore difficult and overfluorinated products are common. However, some reports have shown the ability to favour monofluorination over difluorination by carefully modifying the reaction conditions. ${ }^{40,52} \mathrm{~A}$ further issue is that $\mathrm{C}-\mathrm{H}$ oxygenation side-products can be formed when carboxylic acid additives are required in the catalytic system or when nucleophilic sources are combined with hypervalent iodine reagents bearing oxygenated substituents,.

As elegant as the $\mathrm{C}-\mathrm{H}$ fluorination approach can be, it strongly relies on state-ofthe-art methodologies that require the full consumption of starting material, easy purification of the product and simple scale-up. Yields of isolated products rather than yields determined by ${ }^{19} \mathrm{~F}-\mathrm{NMR}$ should be used to assess the synthetic utility of a protocol. C-H fluorination scale-up processes have emerged ${ }^{241}$ and future advances should consider safety issues, particularly when HF and its derivatives are employed. Cost and atom economy [G] represent important parameters as the field is dominated by reagents that suffer from low atom economy and/or relatively high costs, such as Selectfluor ${ }^{\circledR}, \mathrm{NFSI}$ and $\mathrm{Ag}$ fluorides. In the case of ${ }^{18} \mathrm{~F}$-radiolabelling, operational simplicity and automation are paramount as the ultimate aim is to change clinical practice with the availability of readily automated protocols. 


\section{[H2] Trifluoromethylation}

Each trifluoromethylation method has its own limitations in terms of substrate scope, side reactions and potential for scaling up. The coupling reaction with $\mathrm{CuCF}_{3}$ complex can incorporate a $\mathrm{CF}_{3}$ group into various molecules and may be easily scaled up; however, reaction selectivity can decrease as the electronic nature of the substrate is altered. For example, although aryl chlorides are usually inert and remain intact in coupling reactions, electron-deficient aryl chlorides can be trifluoromethylated. Therefore, attention must be paid to selectivity when substrates contain an active $\mathrm{C}-\mathrm{I}$ or $\mathrm{C}-\mathrm{Br}$ bond and a supposedly [Au:OK? yes] inert $\mathrm{C}-\mathrm{Cl}$ bond Small-scale radical trifluoromethylation reactions have found widespread use in drug and agrochemical development, although when scaling up radical reactions, issues of safety and reaction selectivity should be careful considered as radicals are highly reactive and radical reactions are often exothermic processes. Lowering concentrations or adding reagents slowly can help reduce risk for large-scale radical reactions [Au:OK? yes]. Nucleophilic trifluoromethylation is usually limited to the synthesis of $\alpha-\mathrm{CF}_{3}$ amines, alcohols and ketones; further, reaction temperatures should be kept low in the synthesis of $\alpha-\mathrm{CF}_{3}$ ketones to avoid doubletrifluoromethylation and the formation of tertiary alcohols [Au:OK? yes, NOTE: "tertiary" is added before alcohol]. Finally, electrophilic trifluoromethylation is limited to electron-rich molecules such as enolates and enamines, which can be generated in situ or prepared in advance. If substrates or trifluoromethylation reagents are not reactive enough, the use of Lewis acids may be necessary.

\section{[H2] Difluoromethylation}

Carbon difluoromethylation can introduce a $\mathrm{CF}_{2} \mathrm{H}$ group at an early-stage or latestage and has undoubtedly facilitated the design and discovery of difluoromethylated functional molecules with novel or improved properties. However, some problems must be addressed in terms of both chemistry and practical applications. The control of stereoselectivity at the difluoromethylated $s p^{3}$-carbon mainly depends on 32 / 62
Formatted: Font color: Blue

Formatted: Font color: Red

Formatted: Font color: Red 
diastereoselective nucleophilic difluoromethylation reactions with either chiral substrates or chiral reagents, ${ }^{22}$ whereas enantioselective difluoromethylation remains a challenging synthetic task. ${ }^{46}$ Further, $\cdot \mathrm{CF}_{2} \mathrm{H}$ prefers reacting with relatively electrondeficient $\pi$-systems including heteroarenes owing to its nucleophilic profile; therefore, direct radical $\mathrm{C}-\mathrm{H}$ difluoromethylation of electron-rich arenes is usually problematic although reports have shown that the use of electrophilic surrogates such as $\mathrm{CF}_{2} \mathrm{SO}_{2} \mathrm{Ph}$ and $\cdot \mathrm{CF}_{2} \mathrm{Cl}$ can overcome this limitation. ${ }^{171,172}$

The practical application of difluoromethylation methods by the medicinal chemistry community is highly dependent on the commercial availability and cost of difluoromethylation reagents and catalysts, and the simplicity of the experimental procedures. At present, popular carbon-difluoromethylation reagents include the commercially available $\mathrm{TMSCF}_{2} \mathrm{H},{ }^{130} \quad \mathrm{PhSO}_{2} \mathrm{CF}_{2} \mathrm{H},{ }^{22} \quad\left(\mathrm{HCF}_{2} \mathrm{SO}_{2}\right)_{2} \mathrm{Zn},{ }^{165}$ $\left(\mathrm{HCF}_{2} \mathrm{SO}_{2}\right)_{2} \mathrm{Na}^{137}$ and 2- $\mathrm{BTSO}_{2} \mathrm{CF}_{2} \mathrm{H}_{2}{ }^{225}$ and the corresponding difluoromethylation protocols have been proved to be reproducible in many studies. ${ }^{30,126-129}$ However, most newly developed difluoromethylation methods are not practical for industrial application, owing to the high cost of reagents and metal catalysts. ${ }^{2}$ To reduce the cost, recent research has begun to focus on the use of difluoromethylation reagents closely related to the fluorine industry ${ }^{145,153,161,168,169}$ and inexpensive base-metal catalysts such as $\mathrm{Ni}$ and Fe. ${ }^{152-157}$

\section{[H2] Monofluoromethylation}

Although electrophilic $\mathrm{C}-\mathrm{H}$ monofluoromethylation provides a means for the $\mathrm{a}$ monofluoromethylation of 1,3-dicarbonyl systems, there is limited evidence to suggest that these processes will translate to broader collections of carbon nucleophiles. Challenges of translating this approach to other systems include competitive $O$ monofluoromethylation, which can represent the predominant product, and an intolerance to even mildly nucleophilic functional groups. Efforts are required to improve functional group compatibility and scope. The demonstration of radical $\mathrm{C}-\mathrm{H}$ monofluoromethylation of heterocycles represents a critically important advance, 
although the adoption of this process for medicinal chemistry purposes or further expansion of substrate scope has yet to be described. The development of reactions involving the monofluoromethyl radical and, in particular, the use of this process for late stage functionalization of heterocycles through Minisci-like processes requires further exploration to establish this strategy as a dependable tool for lead drug modification [Au:OK? yes].

\section{[H1] OUTLOOK}

The number of fluorine-containing drug candidates is steadily increasing ${ }^{2-6}$ and the selective fluorination and fluoroalkylation of organic molecules will continue to play an important role in the drug discovery and large-scale production of pharmaceuticals. Remarkable advances have been made in synthetic organofluorine chemistry during the past two decades, among which $\mathrm{C}-\mathrm{H}$ fluorination and fluoroalkylation represent frontiers in the field. ${ }^{242,243}$ Ideal fluorination and fluoroalkylation methods should enable precise $\mathrm{C}-\mathrm{H}$ fluorination and fluoroalkylation in a safe, practical, cost-effective and environmentally benign manner. ${ }^{2}$ However, owing to cost considerations, the industry still favours classical fluorination methods in large-scale productions, such as the use of anhydrous HF, F2, Freons or Halons, and innovative new methods are often only used in small scale synthesis, for example in drug discovery. As a result, it seems paradoxical that, although synthetic fluorine chemistry has been flourishing in academia during the past decade, the pharmaceutical industry still calls for action to develop practical fluorination and fluoroalkylation methods for large-scale synthesis ${ }^{2}$. The synthesis of ${ }^{18} \mathrm{~F}$-labelled radiotracers is in small scale production, although ${ }^{18} \mathrm{~F}$ radiochemistry faces other challenges such as dealing with the short half-life of ${ }^{18} \mathrm{~F}$ and the necessity for translation onto automated platforms for clinical applications. To address the above call for action, ${ }^{2}$ new fluorination and fluoroalkylation methods and manufacturing technologies need to be developed for practical synthesis by choosing cost-effective reagents and/or catalysts in an environmentally responsible way. Readily 
available fluorine sources such as simple inorganic fluorides and organic fluorides, and non-noble metal catalysts or organocatalysts should be preferentially used in synthesis. These new methods need to tolerate heterocycles and other common functional groups and give the desired fluorinated products in high yields for ease of purification.

Asymmetric synthesis of organofluorine compounds is also of high importance and should receive special attentionfeceived special attention.

In the next decade, we expect that [Au:OK? ves] the number of transition metalcatalyzed fluorination and fluoroalkylation reactions will continue to grow, owing to their diverse reactivity and tunable selectivity. Fluorination and fluoroalkylation reactions involving radical intermediates, especially visible light-promoted catalytic reactions, are expected to further flourish, and these radical reactions should find industrial applications with the development of new reaction engineering, techniques. The design and development of new fluorination and fluoroalkylation reagents will add new tools to the synthetic toolbox and enable the construction and introduction of other useful fluorinated functionalities such as $\mathrm{SF}_{5}, \mathrm{OCF}_{3}$ and $t-\mathrm{C}_{4} \mathrm{~F}_{9}$. The incorporation of synthetic biology techniques into synthetic fluorine chemistry, such as the use of enzymatic fluorination and fluoroalkylation schemes, could pave the way for environmentalfriendly fluorinations and fluoroalkylations. We also anticipate that electrochemistry will play a major role in facilitating the late-stage installation of a fluorine substituent. ${ }^{18} \mathrm{~F}$ labelling technologies are also expected to reach new heights with the development of novel PET imaging agents. More generally, it is likely that the field will expand with further development of site-selective functionalization of biological molecules, such as the functionalization of proteins with fluorine or fluoroalkyl motifs. ${ }^{244}$ The combination of synthetic fluorine chemistry with artificial intelligence (AI) might lead to exciting opportunities for the smart synthesis of organofluorine compounds. Al would be used to design the reaction schemes, optimize reaction conditions, and control organic synthesis robots to develop novel fluorinated materials.

Formatted: Font color: Blue

Formatted: Font color: Red

Formatted: Font color: Blue 
In addition to their use in the pharmaceutical industry, selective fluorination and fluoroalkylation reactions find important applications in the agrochemical industry. The production scale of agrochemicals for crop protection is usually larger than that of pharmaceuticals; however, agrochemicals are much more cost-sensitive. As a result, cost-effective fluorination and fluoroalkylation methodsare more likely to be used in the large-scale production of crop protection agents. Further, fluorination and fluoroalkylation reactions promise to find wide applications in the development of new fluorinated functional materials, such as fluorinated liquid crystals, fluorinated antifingerprint coatings and fluorinated plastics and rubbers, among others. The development of new fluorination and fluoroalkylation methods will also likely stimulate innovations in fluorinated refrigerants and fluorinated polymers, which have great importance in our everyday lives.

\section{ACKNOWLEDGEMENTS}

This work is partially supported by the National Key Research and Development Program of China (2016YFB0101200), the National Natural Science Foundation of China (21632009, 21421002, 21672242, 21971252 and 21991122), Key Programs of the Chinese Academy of Sciences (KGZD-EW-T08), the Key Research Program of Frontier Sciences of CAS (QYZDJ-SSW-SLH049), Shanghai Science and Technology Program (18JC1410601), and the Youth Innovation Promotion Association CAS (2019256). RB acknowledges support from a Natural Sciences and Engineering Research Council (NSERC) of Canada Discovery Grant (2019-06368) and MM was supported by a NSERC CGSM award. The authors also thank the European Research Council (Grant agreements 832994 and 789553) for financial support.

\section{Competing interests}

The authors declare no competing interests. 
929 Supplementary information

930 Supplementary information is available for this paper at

931 https://doi.org/10.1038/s415XX-XXX-XXXX-X

932

37 / 62 


\section{Figure legends}

Figure 1. An outline of synthetic organofluorine chemistry. A Historical developments in organofluorine chemistry. This figure briefly describes major landmarks in the development of organofluorine chemistry. The boxes in yellow show Nobel Prize-winning discoveries. B Overview of fluorination, trifluoromethylation, difluoromethylation, and monofluoromethylation, as discussed in this Primer. This is an overview of state-of-the-art strategies that are suitable for late-stage modification of complex organic molecules. Both $\mathrm{C}-\mathrm{H}$ functionalization and transformations of prefunctionalized substartes are covered. 5-FU, 5-fluorouracil; PTFE, polytetrafluoroethylene; $\mathrm{TMSCF}_{3}$, trifluoromethylsilane (also known as RuppertPrakash reagent) [Au:OK? yes].

Fig. 2 The incorporation of fluorine via $\mathbf{C}-\mathbf{H}$ activation. A Two main modes of activation for $\mathrm{C}-\mathrm{H}$ fluorination; transition metal (TM)-mediated catalysis and a radicalmediated strategy. The carbon-centred radicals are commonly formed via hydrogen abstraction by heteroatom radicals or high-valent metal-oxo intermediates.[ [Au: Could you very briefly mention how the radical is formed? yes] B The proposed catalytic cycle for Pd"-catalyzed C-H fluorination from high-valent metal species. DG, directing group; $\mathrm{F}^{+}$source, electrophilic fluorine source; $\mathrm{F}^{-}$source, nucleophilic fluorine source; [O], oxidant; M, metal; Lm, ligand(s).

Fig. 3 Main Pd-catalyzed and Cu-catalyzed C-H fluorination protocols. A Pd or Cu-catalyzed $\mathrm{C}\left(s p^{2}\right)-\mathrm{H}$ functionalization. Potential fluorine sources and directing groups (DG) are shown for both Pd-catalyzed and Cu-catalyzed reactions. [Au:OK? yes] B Pd-catalyzed $\mathrm{C}\left(s p^{3}\right)-\mathrm{H}$ functionalization. The reactions are achieved through

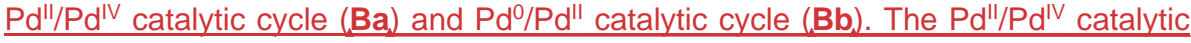
cycle is applicable for a variety of substatres with a proper directing group, and potential fluorine sources and representative directing groups are shown. The $\mathrm{Pd}^{0} / \mathrm{Pd}^{\prime \prime}$ catalytic cycle is limited to allylic compounds and proceeds under the control of a ligand. [Au: Can you expand the description of the figure slightly - can you state what
Formatted: Font color: Blue

Formatted: Font color: Red

Formatted: Font color: Blue
Formatted: Font: Not Bold, Font color: Blue
Formatted: Font: Not Bold
Formatted: Font color: Red

\begin{tabular}{l} 
Formatted: Font color: Red \\
\hline Formatted: Font color: Blue \\
\hline Formatted: Font: Bold, Font color: Blue \\
\hline Formatted: Font color: Blue \\
\hline Formatted: Font: Bold, Font color: Blue \\
\hline Formatted: Font color: Blue \\
\hline
\end{tabular}


is being shown in $\mathrm{Ba}$ and $\mathrm{Bb}$ ? Do the fluorine sources and DGs and ligands refer to schemes shown in $\mathrm{Ba}$ and $\mathrm{Bb}$ ? Yes, we have expanded the description] DG, directing group; EWG, electron-withdrawing group; K2.2.2, Kryptofix ${ }^{\circledR} 222, \mathrm{NFSI}, \mathrm{N}$ fluorosulfonamides; $\mathrm{NMO}, \mathrm{N}$-methylmorpholine $\mathrm{N}$-oxide.

Fig. 4 Main C-H fluorination protocols via carbon-centred radical intermediates.

A C-H fluorination via non-photocatalytic reactions. The above scheme is a general scheme, catalytic systems for use with electrophilic fluorine are shown on the left, catalytic systems for use with neucleophilic fluorine are shown on the right [Au: Can you expand the figure legend to better describe what is being shown here? For example that the above scheme is a general scheme, catalysts for use with electrophilic fluorine are shown on the left, catalysts for use with neutrophilic fluorine are shown on the right? yes, we have expanded the description] B C-H fluorination directed by a proximal functional group. Typical directing groups and catalysts are shown below. C C-H fluorination via photocatalytic reactions. cat., catalytic; FG, proximal functional group; NFSI, N-Fluorobenzenesulfonimide; NDHPI, N,N-dihydroxypyromellitimide; NHPI, N-hydroxyphthalimide; TMP, tetrakis[2,4,6(trimethyl)phenyl]porphyrin; TNP, 5,10,15,20-tetra-naphtyl-porphyrin; TPFPP, 5,10,15,20-Tetrakis(pentafluorophenyl)porphyrin;. ${ }^{25}$

Fig. 5 General overview of trifluoromethylation methods. A Transition-metalcatalyzed trifluoromethylation. Ba Radical trifluoromethylation of alkenes. Bb Radical trifluoromethylation of $s p^{3}$-carbon atoms. C Nucleophilic trifluoromethylation of unsaturated systems. D Electrophilic trifluoromethylation. Ea C-H trifluoromethylation using " $\mathrm{CF}_{3}$ " sources. Eb C-H trifluoromethylation using $\mathrm{CuCF}_{3}$ source. Fa Asymmetric trifluoromethylation using " $\mathrm{CF}_{3}{ }^{\prime}$ " [Au: Can you clarify $\mathrm{CF}_{3}{ }^{\square}, \mathrm{CF}_{3}$, anion] synthons. Fb Asymmetric trifluoromethylation using " $\mathrm{CF}_{3}{ }^{\circ "}$ or " $\mathrm{CF}_{3}{ }^{+"}$ synthons. cat. ${ }^{*}$, catalyst; Halo, halogen; OTf, trifluromethanesulfonate. [Au:OK? yes]

Fig. 6 Example trifluoromethylation protocols. A Transition-metal-catalyzed or mediated cross coupling. Both aryl and alkyl substrates can act as the coupling
Formatted: Font color: Red

Formatted: Font color: Blue
Formatted: Font color: Red

Formatted: Font color: Blue

Formatted: Font color: Red

Formatted: Font color: Red, Subscript

Formatted: Font color: Red, Superscript

Formatted: Font color: Red

Formatted: Font color: Red 
partners. B Radical trifluoromethylation can occur via the attack of a $\mathrm{CF}_{3}{ }^{\cdot}$ radical on an unsaturated bond $(\mathbf{B a})$ or via the transfer of a $\mathrm{CF}_{3}$ group to a radical $(\mathbf{B b})$. Proposed mechanisms are shown in (Bc). [Au: Can you mention Bc in the figure legend? Yes, we have mentioned Bc] C Nucleophilic trifluoromethylation. Trifluoromethyltrimethylsilane $\left(\mathrm{TMSCF}_{3}\right)$ is one of the most commonly used reagents. D Electrophilic trifluoromethylation. This protocol may have limited application because the use of active substrates is usually required. E C-H trifluoromethylation. The activation of a $\mathrm{C}-\mathrm{H}$ bond is usually achieved by the insertion of a metal (Ea) or by radical-mediated homolysis of the $\mathrm{C}-\mathrm{H}$ bond $(\mathbf{E b})$. F Asymmetric catalytic trifluoromethylation. This method includes nucleophilic (Fa), electrophilic (Fb) and radical (Fc) reactions. DMF, dimethylformamide; TBAF, tetra-n-butylammonium fluoride; TfO-, trifluoromethanesulfonate; THF, tetrahydrofuran; MS, molecular sieve: rt, reaction timeroom temperature. [Au: OK? NO, rt stands for room temperature. What does MS stand for in this figure? molecular sieve].

Fig. 7 Main protocols of introducing $\mathrm{CF}_{2} \mathrm{H}$ at $s p^{3}$-carbon centers. The general scheme for each protocol is given in the round rectangle; and the specific examples are shown below the round rectangle. A, nucleophilic addition of difluoromethyl anion or its equivalent $\left(\mathrm{FG}-\mathrm{CF}_{2}{ }^{-}\right)$to unsaturated systems. In the first example, $\mathrm{TMSCF}_{2} \mathrm{H}$ is
Formatted: Font color: Blue

Formatted: Font: Bold, Font color: Blue

Formatted: Font color: Red

Formatted: Font color: Red

Formatted: Font color: Blue

Formatted: Font color: Blue

Formatted: Font color: Red

Formatted: Font color: Red 
1017

normally activated by a fluoride salt; in the second example, $\mathrm{PhSO}_{2} \mathrm{CF}_{2} \mathrm{H}$ is activated by a base such as LiHMDS. Electron-deficient alkenes are also suitable substrates. B, nucleophilic substitution of alkyl electrophiles with a difluoromethyl anion, mainly catalyzed by copper through a $\mathrm{Cu}^{\prime} / \mathrm{Cu}^{\prime \prime} / \mathrm{Cu}^{\prime \prime \prime}$ catalytic cycle. C, addition of difluoromethyl radical to alkenes. After its addition to alkenes, hydrogen abstraction, further functionalization, and further cyclization can occur as the subsequent reaction. $\underline{D}$, difluoromethylation of the $\mathrm{C}-\mathrm{H}$ bonds of carbon acids with difluorocarbene. Reaction with $\mathrm{TMSCF}_{2} \mathrm{Br}$ proceeds in the presence of a base such as tBuOK. E, difluoromethylation of non-activated $\mathrm{C}-\mathrm{H}$ bonds with nucleophilic difluoromethylation reagents catalyzed by copper through a $\mathrm{Cu}^{\prime} / \mathrm{Cu}^{\prime \prime} / \mathrm{Cu}^{\prime \prime \prime}$ catalytic cycle. The carbon cantered radical is formed through hydrogen abstraction of the $\mathrm{C}-\mathrm{H}$ bond by a heteroatom radical such as $\mathrm{N}$-radical that is generated through the cleavage of the $\mathrm{N}$ Cl bond. FG, functional group; EWG, electron-withdrawing group; bbbpy, 4,4'-di-tertbutyl-2,2'-bipyridine; bphen, bathophenanthroline; bpy, 2,2'-bipyridine; DMPU, N, $\mathrm{N}^{\prime}$ dimethylpropyleneurea; TCICA, trichloroisocyanuric acid; tpy, 2,2':6',2"-terpyridine.

[Au: What does the blue colouration of bonds depict in this figure? See the [Au: What does the blue colouration of bonds depict in this figure? See the [Au: What does the blue colouration of bonds depict in this figure? See the [Au: What does the blue colouration of bonds depict in this figure? See the [Au: What does the blue colouration of bonds depict in this figure? See the [Au: What does the blue colouration of bonds depict in this figure? See the [Au: What does the blue colouration of bonds depict in this figure? See the [Au: What does the blue colouration of bonds depict in this figure? See the [Au: What does the blue colouration of bonds depict in this figure? See the [Au: What does the blue colouration of bonds depict in this figure? See the [Au: What does the blue colouration of bonds depict in this figure? See the [Au: What does the blue colouration of bonds depict in this figure? See the ameded figures] 
1045 1046 1047 1048 1048 1049 1050 1051 105 1052 1053 1054 1054 1055 1056 1057 1057 1058 1059 1060 1060 1061 1062 1063 1063 1064 1065 1066 1066 1067

Fig. 8 Main protocols of introducing $\mathrm{CF}_{2} \mathrm{H}$ at (hetero)aromatic carbons (A-C) and heteroatoms (D). The general scheme for each protocol is given in the round rectangle: and the examples are shown without round rectangle. A, the general scheme for difluoromethylation of organohalides or ganopseudohalides (such as aryl triflate and aryl diazonium salt) ( $\mathbf{A a}$ ) and typical examples ( $\mathbf{A b}$ and $\mathbf{A c}$ ). In $\mathbf{A b}$, ( $\mathrm{SIPr}) \mathrm{AgCF}_{2} \mathrm{H}_{\text {, }}$ $\mathrm{TMSCF}_{2} \mathrm{CO}_{2}$ Et and $\mathrm{PhCOCF}_{2} \mathrm{H}$ are also suitable difluoromethylation reagents (under Pd- or Cu-catalysis); In Ac, $\mathrm{HCF}_{2} \mathrm{Br} /(\mathrm{TMS})_{3} \mathrm{SiH}$ is also a suitable combination; however, the use of $\mathrm{Ar}-\mathrm{Br}$ rather than $\mathrm{Ar}-\mathrm{Cl}$ is required (under photocatalysis). B, the general scheme for difluoromethylation of organometallic reagents $(\mathrm{Ba})$ and typical examples ( $\mathrm{Bb}$ and $\mathrm{Bc}$ ). In $\mathrm{Bb}, \mathrm{HCF}_{2} \mathrm{Br}$ is also suitable difluoromethylation reagent (react with arylboronic acids under Ni-catalysis); In $\mathrm{Bc}, \mathrm{BrCF}_{2} \mathrm{P}(\mathrm{O})(\mathrm{OEt})_{2}, \mathrm{Ph}_{3} \mathrm{P}^{+} \mathrm{CF}_{2} \mathrm{CO}_{2}{ }^{-}$and $\mathrm{HCF}_{2} \mathrm{Cl}$ are also suitable difluorocarbene sources. C. the general scheme for $\mathrm{C}-\mathrm{H}$ difluoromethylation of aromatic and heteroaromatic compounds (Ca) and typical examples ( $\mathbf{C b}$ and $\mathbf{C c}$ ). In $\mathbf{C b}$, two pathways for $\mathrm{C}-\mathrm{H}$ difluoromethylation are described; in the radical pathway, both difluoromethyl radical $\left(\mathrm{HCF}_{2}{ }^{\circ}\right)$ and its equivalents (FG$\left.\mathrm{CF}_{2^{\circ}}\right)$ can undergo the reaction. The following are also suitable reagents: $\mathrm{HCF}_{2} \mathrm{CO}_{2} \mathrm{H} /[\mathrm{OO}], \quad \mathrm{HCF}_{2} \mathrm{SO}_{2} \mathrm{Cl}, \quad\left[\mathrm{Ph}_{3} \mathrm{P}\left(\mathrm{CF}_{2} \mathrm{H}\right)\right]^{+} \mathrm{Br}, \quad \mathrm{PhSO}_{2} \mathrm{CF}_{2} \mathrm{l}, \quad\left(\mathrm{ClCF}_{2} \mathrm{CO}\right)_{2} \mathrm{O} /[\mathrm{O}]$, $\mathrm{BrCF}_{2} \mathrm{CO}_{2} \mathrm{Et}$. In Cc, two methods (changing reaction conditions and changing electronic nature of the difluoromethyl group) to alter the regioselectivity are described. one is to change reaction conditions, the other is to change electronic nature of the difluoromethyl group (with $\mathrm{PhSO}_{2}$ or $\mathrm{Cl}$ ). D, the general scheme for difluoromethylation of heteroatoms, for a summary of suitable difluorocarbene sources, one can refer REF. 175. [Red], reductant; [O], oxidant; DMAP, 4dimethylaminopyridine; NMP, N-methyl-2-pyrrolidone; PQ, 9,10-phenanthrenequinone 2-Py, 2-pyridyl; SIPr, 1,3-bis(2,6-diisopropylphenyl)imidazolidene; and transition metal catalyst is depicted with blue color.

Fig. 9 Monofluoromethylating reagents and their use in $\mathrm{C}-\mathrm{H}$ monofluromethylation reactions. A Examples of radical, electrophilic and 
nucleophilic monofluoromethylating reagents. B Protocols for electrophilic $\mathrm{C}-\mathrm{H}$ monofluoromethylation of $\mathrm{C}\left(s p^{3}\right)-\mathrm{H}$ bonds. C Protocols for addition of monofluoromethyl groups to heterocycles via radical processes. D The addition of monofluoromethyl surrogates to aromatics. E Addition reactions with monofluoromethyl radical. F A protocol for nucleophilic monofluoromethylation of ketones. NMP, $N$-methyl-2-pyrrolidone.

Fig. 10 Applications of synthetic organofluorine chemistry in life sciences. A. Applications of organofluorine chemistry in the synthesis and modification of drugs, drug candidates, lead compounds and PET imaging agents. A1-A4 represent the application of organofluorine compounds in the different stages of drug development. For a full list of approved fluorinated drugs, one can refer REF. 207. A5-A8 represent imaging agents synthesized using different methods., $-A 1$, an investigational drug; $A 2$. the derivative of an approved drug; $A 3$, an experimental drug; $A 4$, a lead compound, whose $\mathrm{CF}_{3}$-analogue is an approved drug: $\mathrm{A} 5-\mathrm{A} 6$, synthesized via ${ }^{18} \mathrm{~F}-$-fluorination: A7-A8, synthesized via $\left.{ }^{18} \mathrm{~F}\right]$-fluoroalkylation. [Au: You could expand the legend slightly to help readers - for example noting A1-A4 represent drugs, A5-A8 imaging agents? Yes, we have expanded it. Could you mention why these examples were picked? Yes, we have mentioned the reasons. You can also add any details on the applications if there are any examples that are especially noteworthy if you wish.] B. Example applications of organofluorine chemistry in the modification of natural products_(B1), bioactive small molecules_(B3) and biomacromolecules (B2), and in the production of agrochemicals (B4 and B5). B1, the precursor is a sugar-derivative that is synthesized in laborotoary; B2, the precursor is lysozyme, an enzyme contaning six potentially reactive tryptophan (Trp) residues and eight potentially reactive cysteine (Cys) engaged within each other as disulfides. $\mathrm{CF}_{3}$ is selelctively incorporated into the Trp residues. B3, a bioactive molecule that can be used to probe $\beta$-galactosidase; B4, a herbicide; B5, an insecide. For a full list of approved agrochemicals, see REF. 7. - [Au: As above - the legend can be up to
Formatted: Font color: Blue

Formatted: Font: Not Bold

Formatted: Font color: Blue

Formatted: Font: Not Bold

Formatted: Font color: Blue

Formatted: Font: Not Bold

Formatted: Font: Not Bold, Subscript

Formatted: Font: Not Bold

Formatted: Font: Not Bold, Superscript

Formatted: Font: Not Bold

Formatted: Font color: Red

Formatted: Font color: Red

Formatted: Font color: Red

Formatted: Font: Bold

Formatted: Font: Bold

Formatted: Font: Bold

Formatted: Font: Bold

Formatted: Font: Bold

Formatted: Font color: Blue

Formatted: Font color: Blue

Formatted: Subscript

Formatted: Font color: Blue 
1101200 words so feel free to add a sentence about any particularly noteworth 1102 applications. Yes, we have expanded it.]- 
REFERENCES [au: for references that are particularly worth reading, please provide a single bold sentence that indicates the significance of the work. You are welcome to highlight $10-20$

\section{references.]}

1. Cottet, F., Marull, M., Lefebvre, O. \& Schlosser, M. Recommendable routes to trifluoromethyl-substituted pyridine- and quinolinecarboxylic acids. Eur. J. Org. Chem. 2003, 1559-1568 (2003).

2. Caron, S. Where does the fluorine come from? A review on the challenges associated with the synthesis of organofluorine compound. Org. Process Res. Dev. 24, 470-480 (2020).

3. Zhou, Y. et al. Next generation of fluorine-containing pharmaceuticals, compounds currently in Phase II-III clinical trials of major pharmaceutical companies: new structural trends and therapeutic areas. Chem. Rev. 116, 422-518 (2016).

4. Wang, J. et al. Fluorine in pharmaceutical industry: fluorine-containing drugs introduced to the market in the last decade (2001-2011). Chem. Rev. 114, 2432-2506 (2014).

5. Gillis, E. P., Eastman, K. J., Hill, M. D., Donnelly, D. J. \& Meanwell, N. A. Applications of fluorine in medicinal chemistry. J. Med. Chem. 58, 8315-8359 (2015).

6. Swallow, S. Fluorine in medicinal chemistry. Prog. Med. Chem. 54, 65-133 (2015).

7. Ogawa, Y., Tokunaga, E., Kobayashi, O., Hirai, K. \& Shibata, N. Current contributions of organofluorine compounds to the agrochemical industry. iScience 23, art. no. 101467 (2020).

This paper comprehensively summarized fluorinated agrochemicals on market.

8. Bégué, J.-P. \& Bonnet-Delpon, D. Bioorganic and Medicinal Chemistry of Fluorine. (WileyHoboken, 2008).

9. Fluorine in Medicinal Chemistry and Chemical Biology (ed. Ojima, I.) (Wiley-Blackwell, 2009).

10. Fluorine in Pharmaceutical and Medicinal Chemistry: From Biophysical Aspects to Clinical Applications (eds. Gouverneur, V. \& Müller, K.) (Imperial College Press, 2012).

11. Deng, X. et al. Chemistry for positron emission tomography: recent advances in ${ }^{11} \mathrm{C}-$, ${ }^{18} \mathrm{~F}-,{ }^{13} \mathrm{~N}-$, and ${ }^{15} \mathrm{O}-$ labeling reactions. Angew. Chem. Int. Ed. 58, 2580-2605 (2019).

12. O'Hagan, D., Schaffrath, C., Cobb, S. L., Hamilton, J. T. G. \& Murphy, C. D. Biosynthesis of an organofluorine molecule. Nature 416, 279-279 (2002). 
13. Kirk, K. L. Fluorination in medicinal chemistry: methods, strategies, and recent developments. Org. Process Res. Dev. 12, 305-321 (2008).

14. Dumas, J. \& Peligot, E. Ueber den Holzgeist und die verschiedenen ätherartigen Verbindungen, welche er bildet. Ann. Pharm. 15, 1-60 (1835).

15. Prakash, G. K. S. \& Wang, F. Flourishing Frontiers in Organofluorine Chemistry. In Organic Chemistry: Breakthroughs and Perspectives (eds. Ding, K. \& Dai, L.-X.) (Wiley-VCH, 2012).

16. Fluorine: The First Hundred Years (1886-1986) (eds. Banks, R. E., Sharp, D. W. A. \& Tatlow, J. C.) (Elsevier-New York, 1986).

17. Fluorine Chemistry at the Millennium. Fascinated by Fluorine (eds. Banks, R. E.) (Elsevier- Amsterdam, 2000).

18. Hiyama, T. Organofluorine Compounds: Chemistry and Applications. (Springer-New York, 2000).

19. Chambers, R. D. Fluorine in Organic Chemistry. (Blackwell-Oxford, 2004).

20. Uneyama, K. Organofluorine Chemistry. (Blackwell-Oxford, 2006).

21. Kirsch, P. Modern Fluoroorganic Chemistry: Synthesis, Reactivity, Applications (2nd ed.). (Wiley-VCH, 2013).

22. Ni, C., Hu, M. \& Hu, J. Good partnership between sulfur and fluorine: sulfur-based fluorination and fluoroalkylation reagents for organic synthesis. Chem. Rev. 115, 765825 (2015); and the references on fluorination and fluoroalkylation cited therein.

23. In fluorine chemistry, "direct fluorination" was defined as fluorination using $F_{2}$. See REFS ${ }^{16,19-21 .}$

24. Liu, Q., Ni, C. \& Hu, J. China's flourishing synthetic organofluorine chemistry: innovations in the new millennium. Nat. Sci. Rev. 4, 303-325 (2017).

25. Szpera, R., Moseley, D. F. J., Smith, L. B., Sterling, A. J. \& Gouverneur, V. The fluorination of $\mathrm{C}-\mathrm{H}$ bonds: developments and perspectives. Angew. Chem. Int. Ed. 58, 14824-14848 (2019).

This is a consise review on fluorination of $\mathrm{C}-\mathrm{H}$ bonds.

26. Yerien, D. E., Bonesi, S. \& Postigo, A. Fluorination methods in drug discovery. Org. Biomol. Chem. 14, 8398-8427 (2016).

$27 \mathrm{Ni}, \mathrm{C} . \& \mathrm{Hu}, \mathrm{J}$. The unique fluorine effects in organic reactions: recent facts and insights into fluoroalkylations. Chem. Soc. Rev. 45, 5441-5454 (2016). 
28. Sather A. C. \& Buchwald S. L. The evolution of $\mathrm{Pd}^{0} / \mathrm{Pd}^{\prime \prime}$-catalyzed aromatic fluorination. Acc. Chem. Res. 49, 2146-2157 (2016).

29. Champagne, P. A., Desroches, J., Hamel, J. D., Vandamme, M. \& Paquin, J. F. Monofluorination of organic Compounds: 10 years of innovation. Chem. Rev. 115, 9073-9174 (2015).

30. Emerging fluorinated motifs: synthesis, properties, and applications, Vol. 2 (Eds. Ma, J.-A. \& Cahard, D.) (Wiley-VCH, 2020).

31. Olah, G. A. et al. Synthetic methods and reactions. 63.1 Pyridinium poly(hydrogen fluoride) (30\% pyridine-70\% hydrogen fluoride): a convenient reagent for organic fluorination reactions. J. Org. Chem. 44, 3872-3881 (1979).

32. Haufe, G. Triethylamine trishydrofluoride in synthesis. J. Prakt. Chem./Chem.-Ztg. 338 , 99-113 (1996).

33. Okoromoba, O. E., Han, J., Hammond, G. B. \& Xu, B. Designer HF-based fluorination reagent: highly regioselective synthesis of fluoroalkenes and gem-difluoromethylene compounds from alkynes. J. Am. Chem. Soc. 136, 14381-14384 (2014).

34. Hu, W. L., Hu, X. G. \& Hunter, L. Recent developments in the deoxyfluorination of alcohols and phenols: new reagents, mechanistic insights, and applications. Synthesis 49, 49174930 (2017).

35. Liang, S., Hammond, G. B. \& Xu, B. Hydrogen bonding: regulator for nucleophilic fluorination. Chem. Eur. J. 23, 17850-17861 (2017).

36. Baudoux, J. \& Cahard, D. Electrophilic Fluorination with N-F Reagents. in Organic Reactions 1-326 (John Wiley \& Sons, Inc., 2008).

37. Watson, D. A. et al. Formation of $\operatorname{ArF}$ from $\operatorname{LPdAr}(\mathrm{F})$ : catalytic conversion of aryl triflates to aryl fluorides. Science 325, 1661-1664 (2009).

38. Hull, K. L., Anani, W. Q. \& Sanford, M. S. Palladium-catalyzed fluorination of carbonhydrogen bonds. J. Am. Chem. Soc. 128, 7134-7135 (2006).

39. Casitas, A., Canta, M., Solà, M., Costas, M. \& Ribas, X. Nucleophilic aryl fluorination and aryl halide exchange mediated by a Cul/Culll catalytic cycle. J. Am. Chem. Soc. 133, 19386-19392 (2011).

This is a seminal work on copper-catalyzed aromatic fluorination.

40. Truong, T., Klimovica, K. \& Daugulis, O. Copper-catalyzed, directing group-assisted fluorination of arene and heteroarene C-H bonds. J. Am. Chem. Soc. 135, 9342-9345 (2013).

41. Rueda-Becerril, M. et al. Direct C-F bond formation using photoredox catalysis. J. Am. Chem. Soc. 136, 2637-2641 (2014).

42. Ventre, S., Petronijevic, F. R. \& Macmillan, D. W. C. Decarboxylative fluorination of aliphatic carboxylic acids via photoredox catalysis. J. Am. Chem. Soc. 137, 5654-5657 (2015). 
43. Beeson, T. D. \& MacMillan, D. W. C. Enantioselective organocatalytic $\alpha$-fluorination of aldehydes. J. Am. Chem. Soc. 127, 8826-8828 (2005).

44. Wang, X., Lan, Q., Shirakawa, S. \& Maruoka, K. Chiral bifunctional phase transfer catalysts for asymmetric fluorination of $\beta$-keto esters. Chem. Commun. 46, 321-323 (2010).

45. Rauniyar, V., Lackner, A. D., Hamilton, G. L. \& Dean Toste, F. Asymmetric electrophilic fluorination using an anionic chiral phase-transfer catalyst. Science 334, 1681-1684 (2011).

46. Yang, X., Wu, T., Phipps, R. J. \& Toste, F. D. Advances in catalytic enantioselective

46. Yang, X., Wu, T., Phipps, R. J. \& Toste, F. D. Advances in catalytic enantioselective fluorination, mono-, di-, and trifluoromethylation, and trifluoromethylthiolation reactions. Chem. Rev. 115, 826-870 (2015).

47. Pupo, G. et al. Asymmetric nucleophilic fluorination under hydrogen bonding phasetransfer catalysis. Science 360, 638-642 (2018).

This paper provideded an conceptually novel method for asymmetric nucleophilic fluorination.

48. Pupo, G. et al. Hydrogen bonding phase-transfer catalysis with potassium fluoride: Enantioselective synthesis of $\beta$-fluoroamines. J. Am. Chem. Soc. 141, 2878-2883 (2019).

49. Roagna, G. et al. Hydrogen bonding phase-transfer catalysis with ionic reactants: Enantioselective synthesis of $\gamma$-fluoroamines. J. Am. Chem. Soc. 142, 33, 14045-14051 (2020).

50. Neumann, C. N. \& Ritter, T. Late-stage fluorination: fancy novelty or useful tool? Angew. Chem. Int. Ed. 54, 3216-3221 (2015).

51. Furuya, T. et al. Mechanism of C-F reductive elimination from palladium(IV) fluorides. J. Am. Chem. Soc. 132, 3793-3807 (2010).

52. Chan, K. S. L., Wasa, M., Wang, X. \& Yu, J. Q. Palladium(II)-catalyzed selective monofluorination of benzoic acids using a practical auxiliary: A weak-coordination approach. Angew. Chem. Int. Ed. 50, 9081-9084 (2011).

53. Lou, S. J., Xu, D. Q. \& Xu, Z. Y. Mild and versatile nitrate-promoted C-H bond fluorination. Angew. Chem. Int. Ed. 53, 10330-10335 (2014).

54. Lee, S. J., Makaravage, K. J., Brooks, A. F., Scott, P. J. H. \& Sanford, M. S. Coppermediated aminoquinoline-directed radiofluorination of aromatic $\mathrm{C}-\mathrm{H}$ bonds with $\mathrm{K}^{18} \mathrm{~F}$. Angew. Chem. Int. Ed. 131, 3151-3154 (2019).

55. Zhao, S.-B.; Becker, J. J.; Gagné, M. R.; Steric crowding makes challenging Csp ${ }^{3}-\mathrm{F}$ reductive eliminations feasible. Organometallics 30, 3926-3929 (2011).

56. Racowski, J. M., Gary, J. B. \& Sanford, M. S. Carbon(sp³)-fluorine bond-forming reductive elimination from palladium(IV) complexes. Angew. Chem. Int. Ed. 51, 3414-3417 (2012).

57. Zhang, Q., Yin, X. S., Chen, K., Zhang, S. Q. \& Shi, B. F. Stereoselective synthesis of chiral $\beta$-fluoro $\alpha$-amino acids via $\mathrm{Pd}(\mathrm{II})$-catalyzed fluorination of unactivated methylene $\mathrm{C}\left(\mathrm{sp}^{3}\right)-\mathrm{H}$ bonds: scope and mechanistic studies. J. Am. Chem. Soc. 137, 8219-8226 (2015). 
58. Zhu, R. Y. et al. Ligand-enabled stereoselective $\beta-\mathrm{C}(\mathrm{sp} 3)-\mathrm{H}$ fluorination: synthesis of unnatural enantiopure anti- $\beta$-fluoro- $\alpha$-amino acids. J. Am. Chem. Soc. 137, 7067-7070 (2015).

59. Liao, G., Zhang, T., Lin, Z.-K. \& Shi, B.-F. Transition metal-catalyzed enantioselective C$\mathrm{H}$ functionalization via chiral transient directing group strategies. Angew. Chem. Int. Ed. 59, 19773-19786 (2020).

60. Park, H., Verma, P., Hong, K. \& Yu, J. Q. Controlling Pd(IV) reductive elimination pathways enables $\mathrm{Pd}(\mathrm{II})$-catalyzed enantioselective $\mathrm{C}\left(\mathrm{sp}^{3}\right)$-H Fluorination. Nat. Chem. 10, 755762 (2018).

61. Braun, M. G. \& Doyle, A. G. Palladium-catalyzed allylic C-H fluorination. J. Am. Chem. Soc. 135, 12990-12993 (2013).

62. Rueda-Becerril, M. et al. Fluorine transfer to alkyl radicals. J. Am. Chem. Soc. 134, 40264029 (2012).

63. Bloom, S. et al. A polycomponent metal-catalyzed aliphatic, allylic, and benzylic fluorination. Angew. Chem. Int. Ed. 51, 10580-10583 (2012).

64. Danahy, K. E., Cooper, J. C. \& Van Humbeck, J. F. Benzylic fluorination of azaheterocycles induced by single-electron transfer to selectfluor. Angew. Chem. Int. Ed. 130, 5228-5232 (2018).

65. Meanwell, M., Nodwell, M. B., Martin, R. E. \& Britton, R. A Convenient late-stage fluorination of pyridylic $\mathrm{C}-\mathrm{H}$ Bonds with $\mathrm{N}$-fluorobenzenesulfonimide. Angew. Chem. Int. Ed. 55, 13244-13248 (2016).

66. Bower, J. K., Cypcar, A. D., Henriquez, B., Stieber, S. C. E. \& Zhang, S. C(sp $\left.{ }^{3}\right)-H$ fluorination with a copper(II)/(III) redox couple. J. Am. Chem. Soc. 142, 8514-8521 (2020).

67. Liu, W. et al. Oxidative aliphatic C-H fluorination with fluoride ion catalyzed by a manganese porphyrin. Science 337, 1322-1325 (2012).

68. Liu, W. \& Groves, J. T. Manganese-catalyzed oxidative benzylic C-H fluorination by fluoride ions. Angew. Chem. Int. Ed. 52, 6024-6027 (2013).

69. Huang, X. et al. Late stage benzylic C-H fluorination with $\left[{ }^{18} \mathrm{~F}\right]$ fluoride for PET imaging. $J$. Am. Chem. Soc. 136, 6842-6845 (2014).

70. Groendyke, B. J., Abusalim, D. I. \& Cook, S. P. Iron-catalyzed, fluoroamide-directed C-H fluorination. J. Am. Chem. Soc. 138, 12771-12774 (2016).

71. Xia, J. B., Zhu, C. \& Chen, C. Visible light-promoted metal-free C-H activation: diarylketonecatalyzed selective benzylic mono- and difluorination. J. Am. Chem. Soc. 135, 17494 17500 (2013).

72. Nodwell, M. B. et al. ${ }^{18} \mathrm{~F}$-Fluorination of unactivated C-H bonds in Branched aliphatic amino acids: direct synthesis of oncological positron emission tomography imaging agents. $J$. Am. Chem. Soc. 139, 3595-3598 (2017).

73. Yuan, Z. et al. Site-Selective, Late-Stage $\mathrm{C}-\mathrm{H}^{18} \mathrm{~F}$-fluorination on unprotected peptides for positron emission tomography imaging. Angew. Chem. Int. Ed. 130, 12915-12918 (2018). 
74. Fier, P. S. \& Hartwig, J. F. Selective C-H fluorination of pyridines and diazines inspired by a classic amination reaction. Science 342, 956-960 (2013).

75. Yamamoto, K. et al. Palladium-catalyzed electrophilic aromatic C-H fluorination. Nature 554, 511-514 (2018).

76. Alonso, C., Martinez de Marigorta, E., Rubiales, G. \& Palacios, F. Carbon trifluoromethylation reactions of hydrocarbon derivatives and heteroarenes. Chem. Rev. 115, 1847-1935 (2015).

77. Hu, W.-Q., Pan, S., Xu, X.-H., Vicic, D. A. \& Qing, F.-L. Nickel-mediated trifluoromethylation of phenol derivatives by aryl C-O bond activation. Angew. Chem. Int. Ed. 59, 1607616082 (2020).

78. Meucci, E. A. et al. Nickel(IV)-catalyzed C-H trifluoromethylation of (hetero)arenes. J. Am. Chem. Soc. 141, 12872-12879 (2019).

79. Cho, E. J. et al. The palladium-catalyzed trifluoromethylation of aryl chlorides. Science $\mathbf{3 2 8}$, 1679-1681 (2010). This paper describes the trifluoromethylation of aryl chlorides via a $\mathrm{Pd}(0) / \mathrm{Pd}(\mathrm{II})$ catalytic cycle.

80. Li, G.-B., Zhang, C., Song, C. \& Ma, Y.-D. Progress in copper-catalyzed trifluoromethylation. Beilstein J. Org. Chem. 14, 155-181 (2018).

81. Zeng, Y. et al. Silver-mediated trifluoromethylation-iodination of arynes. J. Am. Chem. Soc. 135, 2955-2958 (2013).

82. Gonda, Z. et al. Efficient copper-catalyzed trifluoromethylation of aromatic and heteroaromatic iodides: the beneficial anchoring effect of borates. Org. Lett. 16, 42684271 (2014)

83. Hu, M., Ni, C. \& Hu, J. Copper-mediated trifluoromethylation of alpha-diazo esters with $\mathrm{TMSCF}_{3}$ : the important role of water as a promoter. J. Am. Chem. Soc. 134, 1525715260 (2012).

84. Chu, L. \& Qing, F.-L. Copper-mediated oxidative trifluoromethylation of boronic acids. Org. Lett. 12, 5060-5063 (2010).

85. Chen, Q.-Y. \& Wu, S.-W. Methyl fluorosulphonyldifluoroacetate; a new trifluoromethylating agent. J. Chem. Soc., Chem. Commun. 705-706 (1989). Methyl fluorosulphonyldifluoroacetate has proved to be an efficient trifluoromethylation reagent and the Cu-mediated trifluoromethylation by using this reagent can be scaled up to kilograms.

86. Clarke, S. L. \& McGlacken, G. P. Methyl fluorosulfonyldifluoroacetate (MFSDA): an underutilised reagent for trifluoromethylation. Chem. Eur. J. 23, 1219-1230 (2017).

87. Xie, Q. \& Hu, J. Chen's reagent: a versatile reagent for trifluoromethylation, difluoromethylenation, and difluoroalkylation in organic synthesis. Chin. J. Chem. 38, 202-212 (2020).

88. Knauber, T., Arikan, F., Roeschenthaler, G.-V. \& Gooßen, L. J. Copper-catalyzed trifluoromethylation of aryl iodides with potassium (trifluoromethyl)trimethoxyborate. Chem. Eur. J. 17, 2689-2697 (2011). 
89. Zanardi, A., Novikov, M. A., Martin, E., Benet-Buchholz, J. \& Grushin, V. V. Direct cupration of fluoroform. J. Am. Chem. Soc. 133, 20901-20913 (2011).

90. Novák, P., Lishchynskyi, A. \& Grushin, V. V. Fluoroform-derived $\mathrm{CuCF}_{3}$ for low-cost, simple, efficient, and safe trifluoromethylation of aryl boronic acids in air. Angew. Chem. Int. Ed. 51, 7767-7770 (2012).

91. Zhang, C.-P. et al. Copper-mediated trifluoromethylation of heteroaromatic compounds by trifluoromethyl sulfonium salts. Angew. Chem. Int. Ed. 50, 1896-1900 (2011).

This trifluoromethylation method has found valuable applications in drug developments.

92. Dai, J.-J. et al. Copper-promoted Sandmeyer trifluoromethylation reaction. J. Am. Chem. Soc. 135, 8436-8439 (2013).

93. Morimoto, H., Tsubogo, T., Litvinas, N. D. \& Hartwig, J. F. A broadly applicable copper reagent for trifluoromethylations and perfluoroalkylations of aryl iodides and bromides. Angew. Chem. Int. Ed. 50, 3793-3798 (2011).

94. Morstein, J., Hou, H., Cheng, C. \& Hartwig, J. F. Trifluoromethylation of arylsilanes with [(phen)CuCF ${ }_{3}$. Angew. Chem. Int. Ed. 55, 8054-8057 (2016).

95. Tomashenko, O. A., Escudero-Adan, E. C., Belmonte, M. M. \& Grushin, V. V. Simple, stable, and easily accessible well-defined $\mathrm{CuCF}_{3}$ aromatic trifluoromethylating agents. Angew. Chem. Int. Ed. 50, 7655-7659 (2011).

96. Paeth, M. et al. $\mathrm{Csp}^{3}-\mathrm{Csp}^{3}$ bond-forming reductive elimination from well-defined copper(III) complexes. J. Am. Chem. Soc. 141, 3153-3159 (2019).

97. Lu, Z. et al. A key intermediate in copper-mediated arene trifluoromethylation, $\left[{ }^{n} \mathrm{Bu}_{4}\right.$ $\mathrm{N}]\left[\mathrm{Cu}(\mathrm{Ar})\left(\mathrm{CF}_{3}\right)_{3}\right]$ : synthesis, characterization, and $\mathrm{C}\left(\mathrm{sp}^{2}\right)-\mathrm{CF}_{3}$ reductive elimination. Angew. Chem. Int. Ed. 58, 8510-8514 (2019).

98. Liu, S. et al. $\mathrm{C}\left(\mathrm{sp}^{3}\right)-\mathrm{CF}_{3}$ reductive elimination from a five-coordinate neutral copper(III) complex. J. Am. Chem. Soc. 142, 9785-9791 (2020).

99. Zhang, C. Application of Langlois' reagent in trifluoromethylation reactions. Adv. Synth. Catal. 356, 2895-2906 (2014).

100. Wu, X., Chu, L. \& Qing, F. L. Silver-catalyzed hydrotrifluoromethylation of unactivated alkenes with $\mathrm{CF}_{3} \mathrm{SiMe}_{3}$. Angew. Chem. Int. Ed. 52, 2198-2202 (2013).

101. Wang, S.-M., Han, J.-B., Zhang, C.-P., Qin, H.-L. \& Xiao, J.-C. An overview of reductive trifluoromethylation reactions using electrophilic ' ${ }^{+} \mathrm{CF}_{3}$ ' reagents. Tetrahedron 71,7949 7976 (2015).

102. Charpentier, J., Früh, N. \& Togni, A. Electrophilic trifluoromethylation by use of hypervalent iodine reagents. Chem. Rev. 115, 650-68 (2015).

103. Iqbal, N., Jung, J., Park, S. \& Cho, E. J. Controlled trifluoromethylation reactions of alkynes through visible-light photoredox catalysis. Angew. Chem. Int. Ed. 53, 539-542 (2014).

104. Merino, E. \& Nevado, C. Addition of $\mathrm{CF}_{3}$ across unsaturated moieties: a powerful functionalization tool. Chem. Soc. Rev. 43, 6598-6608 (2014).

105. Egami, H. \& Sodeoka, M. Trifluoromethylation of alkenes with concomitant introduction of additional functional groups. Angew. Chem. Int. Ed. 53, 8294-8308 (2014).
Formatted: Font color: Blue 
106. Zhu, L., Fang, Y. \& Li, C. Trifluoromethylation of alkyl radicals: breakthrough and challenges. Chin. J. Chem. 38, 787-789 (2020).

107. Prakash, G. K. S. \& Mandal, M. Nucleophilic trifluoromethylation tamed. J. Fluorine Chem. 112, 123-131 (2001).

This paper details the synthetic utilities of a commonly used nucleophilic trifluoromethylation reagent, $\mathrm{TMSCF}_{3}$

108. Prakash, G. K. S. \& Yudin, A. K. Perfluoroalkylation with organosilicon reagents. Chem. Rev. 97, 757-786 (1997).

109. Umemoto, T. \& Ishihara, S. Power-variable electrophilic trifluoromethylating agents. S-, Se-, and Te-(trifluoromethyl)dibenzothio-, -seleno-, and -tellurophenium salt system. J. Am. Chem. Soc. 115, 2156-2164 (1993).

110. Matsnev, A. et al. Efficient access to extended Yagupolskii-Umemoto-Type reagents: triflic acid catalyzed intramolecular cyclization of ortho-ethynylaryltrifluoromethylsulfanes. Angew. Chem. Int. Ed. 49, 572-576 (2010).

111. Wang, X., Truesdale, L. \& Yu, J.-Q. Pd(II)-catalyzed ortho-trifluoromethylation of arenes using TFA as a promoter. J. Am. Chem. Soc. 132, 3648-3649 (2010).

112. Zhang, X.-G., Dai, H.-X., Wasa, M. \& Yu, J.-Q. Pd(II)-catalyzed ortho trifluoromethylation of arenes and insights into the coordination mode of acidic amide directing groups. $J$. Am. Chem. Soc. 134, 11948-11951 (2012).

113. Miura, M., Feng, C.-G., Ma, S. \& Yu, J.-Q. Pd(II)-catalyzed ortho-trifluoromethylation of benzylamines. Org. Lett. 15, 5258-5261 (2013).

114. Chu, L. \& Qing, F.-L. Copper-catalyzed direct C-H oxidative trifluoromethylation of heteroarenes. J. Am. Chem. Soc. 134, 1298-1304 (2012).

115. Shang, M. et al. Exceedingly fast copper(II)-promoted ortho $\mathrm{C}-\mathrm{H}$ trifluoromethylation of arenes using $\mathrm{TMSCF}_{3}$. Angew. Chem. Int. Ed. 53, 10439-10442 (2014).

116. Liu, Z. et al. Copper-catalyzed remote $\mathrm{C}\left(\mathrm{sp}^{3}\right)-\mathrm{H}$ trifluoromethylation of carboxamides and sulfonamides. Angew. Chem. Int. Ed. 58, 2510-2513 (2019).

117. Xiao, H. et al. Copper-catalyzed late-stage benzylic $\mathrm{C}\left(\mathrm{sp}^{3}\right)-\mathrm{H}$ trifluoromethylation. Chem 5, 940-949 (2019).

118. Sarver, P. J. et al. The merger of decatungstate and copper catalysis to enable aliphatic $\mathrm{C}\left(\mathrm{sp}^{3}\right)-\mathrm{H}$ trifluoromethylation. Nature Chem. 12, 459-467 (2020).

119. Zheng, Y. \& Ma, J.-A. Combination catalysis in enantioselective trifluoromethylation. Adv. Synth. Catal. 352, 2745-2750 (2010).

120. Bizet, V., Besset, T., Ma, J.-A. \& Cahard, D. Recent progress in asymmetric fluorination and trifluoromethylation reactions. Curr. Top. Med. Chem. 14, 901-940 (2014).

121. Calvo, R., Comas-Vives, A., Togni, A. \& Katayev, D. Taming radical intermediates for the construction of enantioenriched trifluoromethylated quaternary carbon centers. Angew. Chem. Int. Ed. 58, 1447-1452 (2019).

122. Banik, S. M., Medley, J. W. \& Jacobsen, E. N. Catalytic, asymmetric difluorination of alkenes to generate difluoromethylated stereocenters. Science 353, 51-54 (2016).
Formatted: Font color: Blue, Subscript

Formatted: Font color: Blue 
123. Hu, J., Zhang, W. \& Wang, F. Selective difluoromethylation and monofluoromethylation reactions. Chem. Commun. 7465-7478 (2009).

This is the first comprehensive review on difluoromethylation and monofluoromethylation.

124. Motherwell, W. B., Tozer, M. J. \& Ross, B. C. A convenient method for replacement of the anomeric hydroxy group in carbohydrates by difluoromethyl functionality. J. Chem. Soc., Chem. Commun. 1437-1439 (1989).

125. Sap, J. B. I. et al. Organophotoredox hydrodefluorination of trifluoromethylarenes with translational applicability to drug discovery. J. Am. Chem. Soc. 142, 9181-9187 (2020).

126. Belhomme, M.-C., Besset, T., Poisson, T. \& Pannecoucke, X. Recent progress toward the introduction of functionalized difluoromethylated building blocks onto $\mathrm{C}\left(\mathrm{sp}^{2}\right)$ and $\mathrm{C}(\mathrm{sp})$ centers. Chem. Eur. J. 21, 12836-12865 (2015).

127. Rong, J., Ni, C. \& Hu, J. Metal-catalyzed direct difluoromethylation reactions. Asian J. Org. Chem. 6, 139-152 (2017).

128. Yerien, D. E., Barata-Vallejo, S. \& Postigo, A. Difluoromethylation reactions of organic compounds. Chem. Eur. J. 23, 14676-14701 (2017).

129. Levi, N., Amir, D., Gershonov, E. \& Zafrani Y. Recent progress on the synthesis of $\mathrm{CF}_{2} \mathrm{H}$ containing derivatives. Synthesis 51, 4549-4567 (2019).

130. Zhao, Y., Huang, W., Zheng, J. \& Hu, J. Efficient and direct nucleophilic difluoromethylation of carbonyl compounds and imines with $\mathrm{Me}_{3} \mathrm{SiCF}_{2} \mathrm{H}$ at ambient or low temperature. Org. Lett. 13, 5342-5345 (2011).

This paper introduced $\mathrm{Me}_{3} \underline{\mathrm{SiCF}}_{2} \underline{\mathrm{H}}\left(\mathrm{TMSCF}_{2} \underline{\mathrm{H}}\right)$ as a general and mild direct difluoromethylation reagent.

131. Endo, Y., Ishii, K. \& Mikami, K. Chiral copper-catalyzed enantioselective Michael difluoromethylation of arylidene meldrum's acids with (difluoromethyl)zinc reagents. Tetrahedron 75, 4099-4103 (2019).

132. Zeng, X. et al. Copper-catalyzed decarboxylative difluoromethylation. J. Am. Chem. Soc. 141, 11398-11403 (2019).

133. Zeng, X. et al. Copper-catalyzed, chloroamide-directed benzylic $\mathrm{C}-\mathrm{H}$ difluoromethylation. J. Am. Chem. Soc. 141, 19941-19949 (2019).

134. Gu, Y., Lu, C., Gu, Y. \& Shen, Q. Ligand-controlled · copper-catalyzed · highly · regioselective · difluoromethylation · of · allylic . chlorides/bromides · and . propargyl bromides. Chin. J. Chem. 36, 55-58 (2018).

135. Song, H., Cheng, R., Min, Q.-Q. \& Zhang, X. Decarboxylative and deaminative alkylation of difluoroenoxysilanes via photoredox catalysis: a general method for site-selective synthesis of difluoroalkylated alkanes. Org. Lett. 22, 7747-7751 (2020).

136. Lemos, A., Lemaire, C. \& Luxen, A. Progress in difluoroalkylation of organic substrates by visible light photoredox catalysis. Adv. Synth. Catal. 361, 1500-1537 (2019).

137. He, Z., Tan, P., Ni, C. \& Hu J. Fluoroalkylative aryl migration of conjugated $\mathrm{N}$-arylsulfonylated amides using easily accessible sodium di- and monofluoroalkanesulfinates. Org. Lett. 17, 1838-1841 (2015).

This paper introduced $\mathrm{HCF}_{2} \underline{\mathrm{SO}}_{2} \mathrm{Na}$ as a practical radical difluoromethylation reagent. 
138. Lin, Q.-Y., Ran, Y., Xu, X.-H. \& Qing, F.-L. Photoredox-catalyzed bromodifluoromethylation of alkenes with (difluoromethyl)triphenylphosphonium bromide. Org. Lett. 18, 2419-2422 (2016).

139. Zhang, W. et al. Leaving group assisted strategy for photoinduced fluoroalkylations using N-hydroxybenzimidoyl chloride esters. Angew. Chem. Int. Ed. 58, 624-627 (2019).

140. Meyer, C. F., Hell, S. M., Misale, A., Trabanco, A. A. \& Gouverneur, V. Hydrodifluoromethylationof alkenes with difluoroacetic acid. Angew. Chem. Int. Ed. 58, 8829-8833 (2019).

141. Xie, Q., Zhu, Z., Li, L., Ni, C. \& Hu, J. A general protocol for C-H difluoromethylation of carbon acids with $\mathrm{TMSCF}_{2} \mathrm{Br}$. Angew. Chem. Int. Ed. 58, 6405-6410 (2019).

142. Zhu, J. et al. Carbon-selective difluoromethylation of soft carbon nucleophiles with difluoromethylated sulfonium ylide. Chin. J. Chem. 36, 1069-1074 (2018).

143. Lu, S.-L. et al. Air- and light-stable $S$-(difluoromethyl)sulfonium salts: $C$-selective electrophilic difluoromethylation of $\beta$-ketoesters and malonates. Org. Lett. 20, 69256929 (2018).

144. Duchemin, N., Buccafusca, R., Daumas, M., Ferey, V. \& Arseniyadis, S. A unified strategy for the synthesis of difluoromethyl- and vinylfluoride-containing scaffolds. Org. Lett. 21, 8205-8210 (2019).

145. Zeng, X. et al. Copper-catalyzed deaminative difluoromethylation. Angew. Chem. Int. Ed. 59, 16398-16403 (2020).

146. Fier, P. S. \& Hartwig, J. F. Copper-mediated difluoromethylation of aryl and vinyl iodides. J. Am. Chem. Soc. 134, 5524-5527 (2012).

147. Ferguson, D. M., Malapit, C. A., Bour, J. R. \& Sanford M. S. Palladium-catalyzed difluoromethylation of aryl chlorides and bromides with $\mathrm{TMSCF}_{2} \mathrm{H}$. J. Org. Chem. 84, 3735-3740 (2019).

148. Gu, Y., Leng X. \& Shen Q. Cooperative dual palladium/silver catalyst for direct difluoromethylation of aryl bromides and iodides. Nat. Commun. 5, art. no. 5405 (2014).

149. Lu, C. et al. Palladium-catalyzed difluoromethylation of aryl chlorides and triflates and its applications in the preparation of difluoromethylated derivatives of drug/agrochemical molecules. J. Org. Chem. 83, 1077-1083 (2018).

150. Lu, C., Gu, Y., Wu, J., Gu, Y. \& Shen, Q. Palladium-catalyzed difluoromethylation of heteroaryl chlorides, bromides and iodides. Chem. Sci. 8, 4848-4852 (2017).

151. Pan, F., Boursalian, G. B. \& Ritter, T. Palladium-catalyzed decarbonylative difluoromethylation of acid chlorides at room temperature. Angew. Chem. Int. Ed. 57, 16871-16876 (2018).

152. Xu, L. \& Vicic, D. A. Direct difluoromethylation of aryl halides via base metal catalysis at room temperature. J. Am. Chem. Soc. 138, 2536-2539 (2016).

153. Xu, C. et al. Difluoromethylation of (hetero)aryl chlorides with chlorodifluoromethane catalyzed by nickel. Nat. Commun. 9, art. no. 1170 (2018).

154. Bacauanu, V. et al. Metallaphotoredox difluoromethylation of aryl bromides. Angew. Chem. Int. Ed. 57, 12543-12548 (2018). 
155. Miao, W. et al. Iron-catalyzed difluoromethylation of arylzincs with difluoromethyl 2-pyridyl sulfone J. Am. Chem. Soc. 140, 880-883 (2018).

156. Fu, X.-P., Xiao, Y.-L. \& Zhang, X. Nickel-catalyzed difluoromethylation of arylboronic acids with bromodifluoromethane. Chin. J. Chem. 36, 143-146 (2018).

157. Motohashi, H., Kato, M. \& Mikami, K. Ligand-less iron-catalyzed aromatic cross-coupling difluoromethylation of Grignard reagents with difluoroiodomethane. J. Org. Chem. 84, 6483-6490 (2019).

158. Hori, K., Motohashi, H., Saito, D. \& Mikami, K. Precatalyst effects on Pd-catalyzed crosscoupling difluoromethylation of aryl boronic acids. ACS Catal. 9, 417-421 (2019).

159. Feng, Z., Min, Q.-Q. \& Zhang, X. Access to difluoromethylated arenes by Pd-catalyzed reaction of arylboronic acids with bromodifluoroacetate. Org. Lett. 18, 44-47 (2016).

This paper represents a mechanically novel method for metal catalyzed difluoromethylation.

160. Deng, X.-Y., Lin, J.-H. \& Xiao, J.-C. Pd-catalyzed transfer of difluorocarbene. Org. Lett. 18, 4384-4387 (2016).

161. Feng, Z., Min, Q.-Q., Fu, X.-P., An, L. \& Zhang, X. Chlorodifluoromethane-triggered formation of difluoromethylated arenes catalyzed by palladium. Nat. Chem. 9, 918-923 (2017).

162. Fu, X.-P. et al. Controllable catalytic difluorocarbene transfer enables access to diversified fluoroalkylated arenes. Nat. Chem. 11, 948-956 (2019).

163. Zhu, S.-Q., Liu, Y.-L., Li, H., Xu, X.-H. \& Qing, F.-L. Direct and regioselective C-H oxidative difluoromethylation of heteroarenes. J. Am. Chem. Soc. 140, 11613-11617 (2018).

164. Zhu, S.-Q., Xua, X.-H. \& Qing, F.-L. Silver-mediated oxidative C-H difluoromethylation of phenanthridines and 1,10-phenanthrolines. Chem. Commun. 53, 11484-11487 (2017).

165. Fujiwara, Y. et al. A new reagent for direct difluoromethylation. J. Am. Chem. Soc. 134, 1494-1497 (2012).

This paper developed a practical method for the direct introduction of difluoromethyl group into organic molecules.

166. Dai, P., Yu, X., Teng, P., Zhang, W.-H. \& Deng, C. Visible-light- and oxygen-promoted direct $\mathrm{Csp}^{2}-\mathrm{H}$ radical difluoromethylation of coumarins and antifungal activities. Org. Lett. 20, 6901-6905 (2018).

167. Zhang, W. et al. Direct C-H difluoromethylation of heterocycles via organic photoredox catalysis. Nat. Commun. 11, art. no. 638 (2020).

168. Sakamoto, R., Kashiwagi, H. \& Maruoka, K. The direct C-H difluoromethylation of heteroarenes based on the photolysis of hypervalent iodine(III) reagents that contain difluoroacetoxy ligands. Org. Lett. 19, 5126-5129 (2017).

169. Tung, T. T., Christensen, S. B. \& Nielsen, J. Difluoroacetic acid as a new reagent for direct C-H difluoromethylation of heteroaromatic compounds. Chem. Eur. J. 23, 1812518128 (2017). 
170. Rubinski, M. A., Lopez, S. E. \& Dolbier, W. R. Jr. Direct access to 2-difluoromethyl indoles via photoredox catalysis. J. Fluorine Chem. 224, 80-88 (2019).

171. Su, Y.-M. et al. Visible light-mediated $\mathrm{C}-\mathrm{H}$ difluoromethylation of electron-rich heteroarenes. Org. Lett. 16, 2958-2961 (2014).

172. McAtee, R. C., Beatty, J. W., McAtee, C. C. \& Stephenson, C. R. J. Radical chlorodifluoromethylation: providing a motif for (hetero)arene diversification. Org. Lett. 20, 3491-3495 (2018).

173. Ye, F. et al. Aryl sulfonium salts for site-selective late-stage trifluoromethylation. Angew. Chem. Int. Ed. 58, 14615-14619 (2019).

174. Zhao, H., Herbert, S., Kinzel, T., Zhang, W. \& Shen, Q. Two ligands transfer from Ag to $\mathrm{Pd}$ : en route to $(\mathrm{SIPr}) \mathrm{Pd}\left(\mathrm{CF}_{2} \mathrm{H}\right)(\mathrm{X})$ and its application in one-pot $\mathrm{C}-\mathrm{H}$ borylation/difluoromethylation. J. Org. Chem. 85, 3596-3604 (2020).

175. Ni, C. \& Hu, J. Recent advances in the synthetic application of difluorocarbene. Synthesis 46, 842-863 (2014).

176. Levchenko, K. et al. Copper-catalyzed O-difluoromethylation of functionalized aliphatic alcohols: access to complex organic molecules with an $\mathrm{OCF}_{2} \mathrm{H}$ group. J. Org. Chem. 81, 5803-5813 (2016).

177. Xie, Q. et al. Efficient difluoromethylation of alcohols using $\mathrm{TMSCF}_{2} \mathrm{Br}$ as a unique and practical difluorocarbene reagent under mild conditions. Angew. Chem. Int. Ed. 56, 3206-3210 (2017).

178. Xiao, X. et al. Recent advances in difluoromethylthiolation. Synthesis 52, 197-207 (2020); and references therein.

179. Reichel, M. \& Karaghiosoff, K. Reagents for Selective Fluoromethylation-A challenge in organofluorine chemistry. Angew. Chem. Int. Ed. 59, 12268-12281 (2020).

This is the most comprehensive review on monofluoromethylation.

180. Harry Szmant, H. \& Dudek, J. Relative chloromethylation rates of some aromatic compounds. J. Am. Chem. Soc. 71, 3763-3765 (1949).

181. Meanwell, M. \& Britton, R. Synthesis of heterobenzylic fluorides. Synthesis 50, 12281236 (2018).

182. Parisi, G. et al. Exploiting a "beast" in carbenoid chemistry: development of a straightforward direct nucleophilic fluoromethylation strategy. J. Am. Chem. Soc. 139, 13648-13651 (2017).

183. Shen, X., Ni, C. \& Hu, J. Highly stereoselective and one-pot synthesis of tetra-substituted monofluoroalkenes with aldehydes and fluorobis(phenylsulfonyl)methane. Chin. J. Chem. 31, 878-884 (2013).

184. Koizumi, T., Hagi, T., Horie, Y. \& Takeuchi, Y. Diethyl 1-fluoro-1phenylsulfonylmethanephosphonate, a versatile agent for the preparation of monofluorinated building blocks. Chem. Pharm. Bull. 35, 3959-3962 (1987).

185. Zhu, L., Ni, C., Zhao, Y. \& Hu, J. 1-tert-Butyl-1H-tetrazol-5-yl fluoromethyl sulfone ( $\mathrm{TBTSO} \mathrm{CH}_{2} \mathrm{~F}$ ): a versatile fluoromethylidene synthon and its use in the synthesis of 
monofluorinated alkenes via JuliaKocienski olefination. Tetrahedron 66, 5089-5100 (2010).

186. Shen, X., Zhou, M., Ni, C., Zhang, W. \& Hu, J. Direct monofluoromethylation of O-, S-, N-, and P-nucleophiles with $\mathrm{PhSO}(\mathrm{NTs}) \mathrm{CH}_{2} \mathrm{~F}$ : the accelerating effect of $\alpha$-fluorine substitution. Chem. Sci. 5, 117-122 (2014).

187. Rong, J. et al. Radical fluoroalkylation of isocyanides with fluorinated sulfones by visiblelight photoredox catalysis. Angew. Chem. Int. Ed. 55, 2743-2747 (2016).

188. Tang, X. J. \& Dolbier, W. R. Efficient Cu-catalyzed atom transfer radical addition reactions of fluoroalkylsulfonyl chlorides with electron-deficient alkenes induced by visible light. Angew. Chem. Int. Ed. 54, 4246-4249 (2015).

189. Cao, J. J., Wang, X., Wang, S. Y. \& Ji, S. J. Mn(III)-mediated reactions of 2-isocyanobiaryl with 1,3-dicarbonyl compounds: efficient synthesis of 6-alkylated and 6-monofluoroalkylated phenanthridines. Chem. Commun. 50, 12892-12895 (2014).

190. Olah, G. A. \& Pavlath, A. The investigation of fluoromethylation. Acta Chim. Acad. Sci. Hung. 3, 425 (1953).

191. Olah, G. A. \& Pavlath, A. The preparation of fluoromethanol. Acta Chim. Acad. Sci. Hung. 3, 203-207 (1953).

192. Orr, J. C., Edwards, J. \& Bowers, A. (Syntex Corp.), US3080395, USA 1963.

193. Ding, T. et al. Highly carbon-selective monofluoromethylation of $\beta$-ketoesters with fluoromethyl lodide. Org. Lett. 21, 6025-6028 (2019).

194. Prakash, G. K. S., Ledneczki, I., Chacko, S. \& Olah, G. A. Direct electrophilic monofluoromethylation. Org. Lett. 10, 557-560 (2008).

195. Liu, Y., Lu, L. \& Shen, Q. Monofluoromethyl-substituted sulfonium ylides: Electrophilic monofluoromethylating reagents with broad substrate scopes. Angew. Chem. Int. Ed. 56, 9930-9934 (2017).

196. Nomura, Y., Tokunaga, E. \& Shibata, N. Inherent oxygen preference in enolate monofluoromethylation and a synthetic entry to monofluoromethyl ethers. Angew. Chem. Int. Ed. 50, 1885-1889 (2011).

197. Yang, Y.-D. et al. Cation versus radical: studies on the $\mathrm{C} / \mathrm{O}$ regioselectivity in electrophilic tri-, di- and monofluoromethylations of $\beta$-ketoesters. ChemistryOpen 1, 221-226 (2012).

198. Yang, Y. D., Wang, X., Tsuzuki, S., Tokunaga, E. \& Shibata, N. Studies on the C/Oregioselectivity in electrophilic fluoromethylations of $\beta$-ketoesters based on thermodynamics by ab initio calculations. Bull. Kor. Chem. Soc. 35, 1851-1854 (2014).

199. Raymond, J. I. \& Andrews, L. Matrix reactions of fluorohalomethanes with alkali metals: Infrared spectrum and bonding in the monofluoromethyl radical. J. Phys. Chem. 75, 3235-3242 (1971).

200. Fujiwara, Y. et al. Practical and innate carbon-hydrogen functionalization of heterocycles. Nature 492, 95-99 (2012).

201. Huang, Q. \& Zard, S. Z. Inexpensive radical methylation and related alkylations of heteroarenes. Org. Lett. 20, 1413-1416 (2018). 
202. Ruan, Z. et al. Ruthenium(II)-catalyzed meta $\mathrm{C}-\mathrm{H}$ mono- and difluoromethylations by phosphine/carboxylate cooperation. Angew. Chem. Int. Ed. 56, 2045-2049 (2017).

203. Li, Z. Y. et al. Ruthenium-catalyzed meta-selective C-H mono- and difluoromethylation of arenes through ortho-metalation strategy. Chem. Eur. J. 23, 3285-3290 (2017).

204. Reutrakul, V. \& Rukachaisirikul, V. Fluoromethyl phenyl sulfoxide: highly convenient syntheses of vinyl fluorides and fluoromethylketones. Tetrahedron Lett. 24, 725-728 (1983).

205. Zhao, Y. et al. Copper-catalyzed debenzoylative monofluoromethylation of aryl iodides assisted by the removable (2-Pyridyl)sulfonyl group. ACS Catal. 3, 631-634 (2013).

206. Peters, D. \& Miethchen, R. Symptoms and treatment of hydrogen fluoride injuries. $J$. Fluorine Chem. 79, 161-165 (1996).

207. Inoue, M., Sumii, Y. \& Shibata, N. Contribution of organofluorine compounds to pharmaceuticals. ACS Omega 5, 10633-10640 (2020).

This paper comprehensively summarized fluorinated pharmaceuticals on market.

208. Halperin, S. D., Fan, H., Chang, S., Martin, R. E. \& Britton, R. A convenient photocatalytic fluorination of unactivated C-H bonds. Angew. Chem. Int. Ed. 53, 4690-4693 (2014).

209. Yale, H. L. The trifluoromethyl group in medical chemistry. J. Med. Chem. 1, 121-133 (1958).

210. Meanwell, N. A. Fluorine and fluorinated motifs in the design and application of bioisosteres for drug design. J. Med. Chem. 61, 5822-5880 (2018).

211. Estrada, A. A. et al. Discovery of highly potent, selective, and brain-penetrable leucinerich repeat kinase 2 (LRRK2) small molecule inhibitors. J. Med. Chem. 55, 9416-9433 (2012).

212. Jurica, E. A. et al. Discovery of pyrrolidine-containing GPR40 agonists: stereochemistry effects a change in binding mode. J. Med. Chem. 60, 1417-1431 (2017).

213. Trump, L. et al. Late-Stage ${ }^{18} \mathrm{~F}$-Difluoromethyl labeling of $\mathrm{N}$-heteroaromatics with high molar activity for PET imaging. Angew. Chem. Int. Ed. 58, 13149-13154 (2019).

214. Kuttruff, C. A., Haile, M., Kraml, J. \& Tautermann C. S. Late-stage functionalization of drug-like molecules using Diversinates. ChemMedChem 13, 983-987 (2018).

215. Noisier, A. F. M. et al. Late-stage functionalization of histidine in unprotected peptides. Angew. Chem. Int. Ed. 58, 19096-19102 (2019).

216. Jiang, J. et al. Late-stage difluoromethylation leading to a self-immobilizing fluorogenic probe for the visualization of enzyme activities in live cells. Chem. Commun. 55, 1500015003 (2019).

217. Smith, J. M., Dixon, J. A., deGruyter, J. N. \& Baran, P. S. Alkyl sulfinates: radical precursors enabling drug discovery. J. Med. Chem. 62, 2256-2264 (2019).

218. Campbell, M. G. et al. Bridging the gaps in ${ }^{18} \mathrm{~F}$ PET tracer development. Nat. Chem. 9 , 1-3 (2017). 
219. Yuan, Z. et al. Electrostatic effects accelerate decatungstate-catalyzed $\mathrm{C}-\mathrm{H}$ fluorination using $\left[{ }^{18} \mathrm{~F}\right]-$ and $\left[{ }^{19} \mathrm{~F}\right] \mathrm{NFSI}$ in small molecules and peptide mimics. ACS Catal. 9, 82768284 (2019).

220. Huiban, M. et al. A broadly applicable $\left[{ }^{18} \mathrm{~F}\right]$ trifluoromethylation of aryl and heteroaryl iodides for PET imaging. Nat. Chem. 5, 941-944 (2013).

221. Ivashkin, $\mathrm{P}$. et al. $\mathrm{CuCF}_{3}$ : a $\left[{ }^{18} \mathrm{~F}\right]$ trifluoromethylating agent for arylboronic acids and aryl iodides. Chem. Eur. J. 20, 9514-9518 (2014).

222. Vanderborn, D. et al. A universal procedure for the $\left[{ }^{18} \mathrm{~F}\right]$ trifluoromethylation of aryl iodides and aryl boronic acids with highly improved specific activity. Angew. Chem. Int. Ed. 53, 11046-11050 (2014).

223. Yang, B. Y., Telu, S., Haskali, M. B., Morse, C. L. \& Pike, V. W. A gas phase route to $\left[{ }^{18} \mathrm{~F}\right]$ fluoroform with limited molar activity dilution. Sci. Rep. 9, art. no. 14835 (2019).

224. Ashworth, S. et al. ${ }^{18} \mathrm{~F}$-Trifluoromethanesulfinate enables direct $\mathrm{C}-\mathrm{H}{ }^{18} \mathrm{~F}$ trifluoromethylation of native aromatic residues in peptides. J. Am. Chem. Soc. 142, 1180-1185 (2020).

225. Zheng, L. \& Berridge, M. S. Synthesis of $\left[{ }^{18} \mathrm{~F}\right]$ fluoromethyl iodide, a synthetic precursor for fluoromethylation of radiopharmaceuticals. Appl. Radiat. Isot. 52, 55-61 (2000).

226. Iwata, R. et al. $\left[{ }^{18} \mathrm{~F}\right] \mathrm{Fluoromethyl} \mathrm{triflate,} \mathrm{a} \mathrm{novel} \mathrm{and} \mathrm{reactive}\left[{ }^{18} \mathrm{~F}\right]$ fluoromethylating agent: Preparation and application to the on-column preparation of $\left[{ }^{18} \mathrm{~F}\right]$ fluorocholine. Appl. Radiat. Isot. 57, 347-352 (2002).

227. Degrado, T. R. et al. Synthesis and evaluation of ${ }^{18} \mathrm{~F}$-labeled choline as an oncologic tracer for positron emission tomography: initial findings in prostate cancer. Cancer Res. 61, 110-117 (2001).

228. Doi, H., Goto, M. \& Suzuki, M. Pd ${ }^{0}$-mediated rapid C- $\left[{ }^{18} \mathrm{~F}\right]$ fluoromethylation by the crosscoupling reaction of a $\left[{ }^{18} \mathrm{~F}\right]$ fluoromethyl halide with an arylboronic acid ester: Novel method for the synthesis of a ${ }^{18} \mathrm{~F}$-labeled molecular probe for positron emission tomography. Bull. Chem. Soc. Jpn. 85, 1233-1238 (2012).

229. Vitaku, E., Smith, D. T. \& Njardarson, J. T. Analysis of the structural diversity, substitution patterns, and frequency of nitrogen heterocycles among US FDA approved pharmaceuticals. J. Med. Chem. 57, 10257-10274 (2014).

230. Ichiishi, N. et al. Protecting group free radical C-H trifluoromethylation of peptides. Chem. Sci. 9, 4168-4175 (2018).

231. Imiołek, M. et al. Selective radical trifluoromethylation of native residues in proteins. J.Am. Chem. Soc. 140, 1568-1571 (2018).

232. O'Neill, B. T. et al. Design and synthesis of clinical candidate PF-06751979: a potent, brain penetrant, $\beta$-site amyloid precursor protein cleaving enzyme 1 (BACE1) inhibitor lacking hypopigmentation. J. Med. Chem. 61, 10, 4476-4504 (2018).

233. Greszler, S. N., Shelat, B. \& Voight, E. A. Enabling synthesis of ABBV-2222, A CFTR corrector for the treatment of cystic fibrosis. Org. Lett. 21, 5725-5727 (2019).

234. Kubota, S. Manufacturing method of pyrazole derivative and intermediate products thereof. JP2017/206453 A (2017). 
235. Fujiwara, T. \& O'Hagan D. Successful fluorine-containing herbicide agrochemicals. J. Fluorine Chem. 167, 16-29 (2014).

236. Okui, S. et al. Pyrazol derivatives, pest control agent comprising the same as active ingredient, and process for producing the same. US7371768 B2 (2008).

237. Sharma, R. \& Aboagye, E. Development of radiotracers for oncology - The interface with pharmacology. Br. J. Pharmacol. 163, 1565-1585 (2011).

238. Everaert, $\mathrm{H}$. et al. Optimal dose of ${ }^{18} \mathrm{~F}-\mathrm{FDG}$ required for whole-body PET using an LSO PET camera. Eur. J. Nucl. Med. Mol. Imaging 30, 1615-1619 (2003).

239. https://www.who.int/medicines/areas/quality_safety/quality_assurance/production/en/

240. Dolbier, W. R. Jr. Guide to fluorine NMR for organic chemists (2nd ed.) (John Wiley \& Sons, 2016)

241. Halperin, S. D. et al. Development of a Direct Photocatalytic C-H Fluorination for the Preparative Synthesis of Odanacatib. Org. Lett. 17, 5200-5203 (2015).

242. Nobile, E., Castanheiro, T. \& Besset, T. Radical-promoted distal C-H functionalization of $\mathrm{C}\left(\mathrm{sp}^{3}\right)$ centers with fluorinated moieties. Angew. Chem. Int. Ed. 60, https://doi.org/10.1002/anie.202009995 (2021).

243. Zhang, F.-G. et al. Remote fluorination and fluoroalkyl(thiol)ation reactions. Chem. Eur. J. 26, 15378-15396 (2021).

244. Josephson, B. et al. Light-driven post-translational installation of reactive protein side chains. Nature 585, 530-537 (2020). 


\section{GLOSSARY}

1713 properties.

Heteroelement: In the periodic table, any element that is not carbon or hydrogen.

Positron emission tomography: A functional imaging technique that uses radiotracers to visualize and measure changes in metabolic processes, and in other physiological activities.

Swarts reaction: A fluorination method that is used to prepare alkyl fluorides from alkyl chlorides or bromides. The typical fluorination reagent is antimony(III) trifluoride in the presence of a catalytic amount of antimony $(\mathrm{V})$ salts.

Balz-Schiemann reaction: A method for the production of aryl fluorides from primary aromatic amine via a diazonium tetrafluoroborate intermediate.

Halex reaction: The nucleophilic substitution reaction between an aryl or alkyl halide with the other halide ions.

Phase transfer catalysis: A process in which the rate of a reaction in a heterogeneous two-phase system is enhanced by the addition of a substance that transfers one of the reactants across the interface between the two phases.

$\mathrm{C}-\mathrm{H}$ bond dissociation energies: It is a measure of the strength of a $\mathrm{C}-\mathrm{H}$ bond, which can be defined as the standard enthalpy change when $\mathrm{C}-\mathrm{H}$ is cleaved by homolysis to give a carbon radical and a hydrogen atom.

Density functional theory: A computational quantum mechanical modelling method to investigate the electronic structure or nuclear structure of atoms, molecules, and the condensed phases.

Heteroatom: Similar to heteroelement, heteroatom refers to any atom that is not carbon atom or hydrogen atom.

Bioisosteres:_Chemical substituents or groups with similar physical or chemical

Formatted: Font color: Blue

Formatted: Font color: Blue

Formatted: Font color: Blue

Formatted: Font color: Blue

Formatted: Font color: Blue

Formatted: Font color: Blue 
1737 Minisci reaction: A nucleophilic radical substitution to an electron deficient aromatic 1738 compound, most commonly involving the introduction of an alkyl group to a nitrogen 1739 containing aromtic heterocycle.

1740 Cryptand: A family of synthetic bicyclic and polycyclic multidentate ligands for a variety 1741 of cations.

1742 Atom economy: The conversion efficiency of a chemical process in terms of all atoms 1743 involved and the desired products produced.
Formatted: Font color: Blue

Formatted: Font color: Blue

Formatted: Font color: Blue 